Título artículo / Títol article:

Kinetic study of the thermal decomposition process of calcite particles in air and $\mathrm{CO}_{2}$ atmosphere

Autores / Autors

Escardino Benlloch, Agustín ; García Ten, Francisco Javier ; Feliu Mingarro, Carlos ; Saburit Llaudis, Alejandro ; Cantavella Soler, Vicente

Revista:

Journal of Industrial and Engineering Chemistry Volume 19, Issue 3, 25 May 2013

Versión / Versió:

Preprint de l'autor

Cita bibliográfica / Cita bibliogràfica (ISO 690):

url Repositori UJI:

http://hdl.handle.net/10234/91792 


\title{
Kinetic study on the thermal decomposition process of calcite particles in air and $\mathrm{CO}_{2}$ atmosphere
}

\author{
A. Escardino*, J. García-Ten, C. Feliu, A. Saburit, V. Cantavella
}

Instituto de Tecnología Cerámica. Asociación de Investigación de las Industrias Cerámicas. Universitat Jaume I. Campus Universitario Riu Sec, 12006 Castellón. Spain.

aescardino@itc.uji.es

\author{
CORRESPONDING AUTHOR FOOTNOTE \\ Tel.: +34964342424 \\ Fax: +34 964342425 \\ e-mail address: agustin.escardino@itc.uji.es
}

\begin{abstract}
The thermal decomposition process of calcite particles $(0.45-3.60 \mathrm{~mm}$ of average diameter), made up of porous agglomerates of very small $\mathrm{CaCO}_{3}$ microcrystals has been studied in the 975-1216 K temperature range under isothermal conditions in presence of mixtures of air and carbon dioxide with different compositions.

An equation, which relates the calcite conversion degree with both reaction time and operating conditions, has been proposed. The equation satisfactorily fits to the experimental results obtained in the entire tested range of particle sizes and of temperatures in all the range of carbon dioxide concentrations studied.
\end{abstract}

KEYWORDS: Kinetics; Particle processing; Calcination; Solid-gas phase reaction. 


\section{Introduction}

\subsection{Background}

One of the methods recently being considered of recovering the carbon dioxide contained in mixtures with other gases consists of $\mathrm{CO}_{2}$ capture using calcium oxide, either by itself or incorporated into different inorganic structures [1-12].

The industrial application of this technology requires the consecutive and cyclical development of two processes: a) carbon dioxide capture by calcium oxide (carbonation); b) thermal decomposition of the resulting calcium carbonate (calcination) in order to regenerate the calcium oxide that is to be used again in the following cycle. The chemical steps of both processes may be represented by means of the following reaction scheme, assuming that a reversible chemical transformation takes place:

$$
\mathrm{CaCO}_{3}(\mathrm{~s}) \leftrightarrow \mathrm{CaO}(\mathrm{s})+\mathrm{CO}_{2}(\mathrm{~g})
$$

In order to optimise the operation of a continuous chemical reactor that would enable both processes to be sequentially developed, it is desirable to have a simple algorithm that allows the degree of transformation progress that develops in each process to be related to the operating conditions (time, temperature, nature and characteristics of the solid used, and $\mathrm{CO}_{2}$ concentration in the gas phase).

Although numerous papers may be found in the literature, some of which are very recent, on the kinetics and mechanism by which both processes develop, discrepancies are still to be noted with regard to the kinetic model used to interpret the experimental results and the rate equation used for the chemical reaction step represented by scheme (1). Moreover, different views are held on how the carbon dioxide concentration in the gas phase quantitatively influences the kinetics of these processes. All researchers agree that the presence of $\mathrm{CO}_{2}$ limits the decomposition reaction rate, but they differ in their understanding of the quantitative form of this dependence.

These discrepancies were already noted by Stanmore and Gilot [13] in 2005 in a review on calcination and carbonation, in which they highlighted 'the need for a realistic model of the process taking place in the particles', a view maintained in subsequent papers [14-17].

In regard to the thermal decomposition of limestone, some researchers propose relatively complex models to interpret the process kinetics. Most have used the 'Grainy Pellet Model' [18-28] or a 'Modified Random Pore Model' [29], assuming that the chemical reaction and $\mathrm{CO}_{2}$ intergrain diffusion steps unfold concurrently in a non-steady-state regime and 
considering the possibility that one of these or both simultaneously control the overall process rate. Some researchers $[18,19]$ assume that each pellet grain decomposes according to the 'Shrinking Unreacted Core Model' [30] and propose a set of differential equations in partial derivatives to interpret the experimental results, using numerical methods to integrate these.

For the chemical decomposition reaction of $\mathrm{CaCO}_{3}$, these researchers use the following rate equation (for the meaning of the symbols please see the Nomenclature section):

$$
r=k \cdot\left(1-c_{Q i}^{s} / c_{Q e}^{s}\right)^{n}
$$

with values for the exponent $n$ ranging from one to two, depending on the value of the temperature considered [18]. Others $[14,17,36,46,54]$ use the same equation, substituting the $\mathrm{Q}\left(\mathrm{CO}_{2}\right)$ molar concentrations by the corresponding pressures.

There are researchers that propose models based on the formation of intermediate metastable products at the chemical reaction interface $[31,32]$ or on a nucleation mechanism (production and growth of nuclei) [33,34]. Finally others interpret the chemical reaction kinetics of the $\mathrm{CaCO}_{3}$ decomposition process and of $\mathrm{CaO}$ carbonation in a $\mathrm{CO}_{2}$ atmosphere, using the rate equations governing the chemisorption-desorption phenomena $[2,15,35,36,37]$.

\subsection{Object of this research}

Given this diversity of views, it was considered that it might be of interest to study both processes in order to attempt to correlate the experimental results using a relatively simple single model, in accordance with the approach adopted by Octave Levenspiel and Albert Einstein ('everything should be made as simple as possible but no simpler') [38].

The calcite decomposition process was studied in air atmosphere, in the 1123-1223 K temperature range in a previous paper [40]. In that study it was observed qualitatively that, for particles with average radii larger than $0.225 \mathrm{~mm}$, the decomposition rate decreased when the average particle radius increased. On the other hand, it was observed that for particles with average radii smaller than $0.225 \mathrm{~mm}$, the process rate was not influenced by the size of the particle. 
In that case, an equation, based on the Grainy Pellet Model, was proposed that fitted well to the experimental data obtained in the range of particle sizes with average radii smaller than $0.225 \mathrm{~mm}$.

To complete that study, this paper presents the results obtained in studying the decomposition process of calcite particles with average radii larger than $0.225 \mathrm{~mm}$, in air atmosphere. Also has been studied the decomposition process of calcite particles in $\mathrm{CO}_{2}$ atmosphere, and in a gas stream comprising different concentrations of air and $\mathrm{CO}_{2}$.

The experiments were carried out in a tubular reactor operating under isothermal conditions. Eight particle size fractions were tested.

A following paper will set out the results obtained in studying the recarbonation process of the $\mathrm{CaO}$ resulting from the thermal decomposition of the calcite particles used in this work.

\section{Materials and experimental procedure}

\subsection{Materials}

The study was conducted with natural calcite particles, supplied by REVERTE S.A., containing $99.4 \%$ (by weight) of calcium carbonate [40].

Eight particle size fractions of this type of calcite (with average radii of $0.225,0.30,0.46$, $0.70,1.025,1.35,1.54$, and $1.80 \mathrm{~mm}$ ) were prepared by dry milling in a hammer mill. The milling product was sieved in a sieve shaker with nested screens. For each particle size fraction used, the average particle radius was calculated from the 'Sauter mean diameter' [39].

Calcite particle bulk density was $2185 \mathrm{~kg} \cdot \mathrm{m}^{-3}$. Given the particle composition, this was equivalent to an initial calcium carbonate content of $21.7 \mathrm{kmol} \cdot \mathrm{m}^{-3}$.

The specific surface area was determined by nitrogen adsorption at $77 \mathrm{~K}$ (BET method) with a MICROMERITICS TRISTAR 3000 gas adsorption analyser. The values obtained were practically independent of particle size, the mean value being $0.62 \pm 0.04 \mathrm{~m}^{2} \mathrm{~g}^{-1}$. This was equivalent to $1354700 \pm 87400 \mathrm{~m}^{2} \cdot \mathrm{m}^{-3}$, taking into account the value of calcite particle bulk density. 
Figures 1 and 2 show the micrographs obtained by scanning electron microscopy (PHILIPS Model XL30) of one of the calcite particles used. The particles are observed to be microcrystal aggregates with a porous structure (aggregate initial porosity was 0.16 ). The microcrystals were smaller than $0.020 \mathrm{~mm}$.

The assays ATD-TG were performed in a thermobalance Mettler model TGA / SDTA 851e.

Figure 1

\section{Figure 2}

\subsection{Experimental assembly}

Thermal decomposition of the calcite particles was carried out in a tubular kiln at controlled temperature. Air, carbon dioxide, or mixtures of both gaseous components could be fed into the kiln at a given flow rate.

Figure 3 schematically illustrates the experimental assembly used. The assembly consists of a tubular kiln in the middle of which was located a refractory steel holder hanging of a singlepan balance. The sample contained in a refractory steel capsule was placed on the holder so that its mass could be continuously measured. The single-pan balance was connected to a computer with the appropriate software to record the corresponding pairs of mass and time values. The single-pan balance was adequately insulated to prevent heat radiated from the reactor can affect the measurements of the sample mass.

\section{Figure 3}

\subsection{Experimental procedure}

All experiments were carried out under isothermal conditions. Some of them were conducted in air atmosphere and others in an atmosphere containing different $\mathrm{CO}_{2}$ concentrations. A sufficiently high gas flow rate was used in all experiments for the $\mathrm{CO}_{2}$ transfer step from the 
calcite particle surface into the gas phase to unfold very quickly and not to influence the overall process rate.

The quantity of powder used in each experiment was $500 \mathrm{mg}$, because it was observed that the experimental results were influenced by sample mass when this was larger. The powder was spread in a very thin layer in the capsule. The sample contained in the capsule was heated at $385 \mathrm{~K}$, in carbon dioxide atmosphere, in an oven for 30 minutes before each experiment. After calibration of the balance, the data logging system was switched on and the capsule, containing the sample, was quickly placed in the kiln. The corresponding pairs of values of sample mass and sample residence time were recorded during every experiment. Each experiment ended when sample mass remained practically constant.

\section{Experimental results}

\subsection{Determination of the calcium carbonate degree of conversion during firing of calcite particles}

The variation of calcium carbonate conversion in the calcite particles with residence time was calculated from the corresponding pairs of values of sample mass and residence time measured in each experiment. It was verified at the end of all the experiments that the calcium carbonate initially contained in the sample had decomposed completely. The calcium carbonate fractional conversion was then calculated from the equation:

$$
X=\frac{\Delta M}{\Delta M_{\infty}}
$$

where $\Delta M$ is mass loss of the sample at a given residence time after experiment commencement and $\Delta M_{\infty}$ is mass loss of the sample at a sufficiently long time to achieve constant weight, when all the calcium carbonate initially present has decomposed.

\subsection{Programming of experiments and presentation of the results obtained}

Several series of experiments were conducted under constant operating conditions, one of the operating variables (temperature, calcite particle size, or composition of the gas phase) being 
modified in each series. Residence time was counted from the moment the capsule with the sample was placed in the reactor, once the reactor had reached the desired reaction temperature. The $X$ values were determined at different residence times.

The experimental data have been plotted (as crosses, squares, circles, triangles, etc.) for each series of experiments, in the figures shown below, as conversion degree of the calcium carbonate contained in the calcite particles versus residence time.

\subsubsection{Experiments conducted in air atmosphere}

The results obtained in the experiments conducted in air atmosphere at five temperatures $(1123,1148,1173,1198$, and $1223 \mathrm{~K})$, with the eight particle size fractions mentioned above, have been plotted in Figures 4 to 8 as indicated above. Each figure corresponds to one studied temperature and contains the experimental results obtained with the eight studied particle size fractions.

\section{Figure 4 \\ Figure 5 \\ Figure 6 \\ Figure 7 \\ Figure 8}

Figures 4 to 8 show that:

a) As expected, the slope of the graphic representation of the experimental data of $X$ versus residence time, for any value of the average particle radius, increases with increasing temperature.

b) For the five studied temperatures, the slope of the graphic representation of $X$ versus residence time decreased as average particle radius increased. This outcome suggests that $\mathrm{CO}_{2}$ diffusion through the particle structure (intergrain diffusion), without or with the chemical reaction, influenced process kinetics in all the studied temperature range. 


\subsubsection{Experiments conducted in the presence of carbon dioxide}

In order to study the influence of the presence of carbon dioxide in the gaseous phase on the decomposition reaction rate of the calcite particles, several experiments were carried out at different temperatures using the particle fraction with an average radius of $0.7 \mathrm{~mm}$, in a gas stream comprising different concentrations of air and $\mathrm{CO}_{2}$ (including only carbon dioxide). The experimental results obtained at the temperatures of 1123, 1150, 1153, 1155, 1158, 1173, 1195, and 1216K, have been plotted in Figures 9 to 14 in the form as it is described above.

Other three series of experiments were conducted at the temperatures of 1098, 1107 and 1113 $\mathrm{K}$ to complete the study about the influence of the temperature on the effective diffusivity and the influence of the percentage of $\mathrm{CO}_{2}$ in the gaseous mixture on the value of $b$ parameter. The results of these experiments have not been plotted in any figure, but in Table 4 are shown the corresponding values of their kinetic parameters calculated following the procedure explained in section 4.3.2.

Figure 9 presents the results obtained in the experiments conducted solely in carbon dioxide. Only the results obtained at the seven highest test temperatures are plotted in this figure, because no decomposition was detected at $1123 \mathrm{~K}$.

\section{Figure 9}

Figures 10 to 14 present the results obtained in the experiments carried out in different concentrations of air and $\mathrm{CO}_{2}$ at test temperatures of $1123,1152,1173,1195$ and $1216 \mathrm{~K}$.

Figure 10

Figure 11

Figure 12

Figure 13

Figure 14

Figures 10 to 14 show qualitatively that, when the $\mathrm{CO}_{2}$ content in the gas phase increased, the graphic representation of $X$ versus residence time shifted towards the $\mathrm{X}$-axis. That is, the rate at which the decomposition process unfolded (which is directly related to the slope of these plots) decreased, as was to be expected, since the calcium carbonate decomposition reaction is reversible. 


\section{Discussion of results}

\subsection{Kinetic model}

The foregoing process was studied in air atmosphere, in the 1123-1223 K temperature range, using particles with average radii smaller than $0.225 \mathrm{~mm}$, in a previous paper [40]. In this range of particle sizes, the process rate was not influenced by the particle radius.

In that case, an equation, based on the Grainy Pellet Model, was proposed that fitted well to the experimental data. That equation was derived, assuming that all grains (microcrystals), in every particle, reacted independently and at the same time and that the chemical reaction step unfolded much more slowly than the $\mathrm{CO}_{2}$ diffusion step into each microcrystal (intragrain diffusion) and among the microcrystals making up the particles (intergrain diffusion) [21-24]. In this case their behaviour coincided with that of the Uniform Conversion Model [25,27]. This assumption is usually valid when the microcrystals and the particle sizes are very small $[22,23,41]$.

However, for the range of average radii involved in the present study $(0.225-1.80 \mathrm{~mm})$, Figures 4 to 8 show that the process rate decreased when the average particle radius increased, at all test temperatures. This behaviour would seem to suggest that, for particle radii larger than $0.225 \mathrm{~mm}$, the process rate was influenced by the $\mathrm{CO}_{2}$ diffusion step among the microcrystals (intergrain diffusion), either by itself or together with the chemical reaction step.

In accordance with these results and taking into account the kinetic model proposed in the previous paper, several possibilities were studied with a view to obtaining a mathematical expression that was simpler than those described in the literature to date and that could satisfactorily represent the experimental results obtained on conducting the process in air atmosphere and in the presence of carbon dioxide.

Making the relevant assumptions and using the methodology described in the Appendix, a series of equations were derived. These equations - which relate calcium carbonate conversion in the calcite particles to reaction time, to the kinetic parameters that depend on temperature (rate constant, equilibrium constant of the chemical reaction step, and intergrain effective diffusivity), to the particle radius, to the initial calcium carbonate molar 
concentration in the particles, and to the $\mathrm{CO}_{2}$ molar concentration in the gaseous phase - were tested with the experimental results.

The equation of the form $X=f(t)$ that best fitted to the experimental results was:

$$
\frac{d X}{d t}=\left(\frac{1}{c_{B}^{0}}\right) \cdot\left[\frac{K_{C}-b \cdot c_{Q}^{G}}{\frac{K_{C}}{k \cdot S_{s p}(1-X)^{1 / 3}}+\frac{r_{S}^{2}}{3 \cdot D_{e}}}\right]
$$

This equation derives from equation (A.14) of the Appendix, setting the exponent $m$ equal to $1 / 3$ in equations (A.2) and (A.3). The value of $m$ proposed is of the same order as that reported by Fujimoto [14] for the $\mathrm{CaCO}_{3}$ decomposition process. Equation (A.14) was derived assuming that the calcite particles had a cubic shape and that the relationship between the molar concentrations of $\mathrm{CO}_{2}$ on both sides of the gas-particle interface was governed by an equilibrium law.

Equation (4) fitted well to the experimental data obtained, at all tested temperatures, in the case of the particles with radii equal to or smaller than $0.460 \mathrm{~mm}$. However, it only fitted well to the experimental results obtained with the particles of average radius equal to or larger than $0.700 \mathrm{~mm}$ up to a conversion degree of 0.75 .

In order to attempt to find an equation that better fitted to the experimental data in the higher stretch of conversion degrees, in the case of the larger studied particles, a modification was introduced in equation (4) based on the fact that, according to the literature $[25,26,28]$, the behaviour of the 'Grainy Pellet Model' is usually intermediate between that of the 'Uniform Conversion Model' and the 'Shrinking Unreacted Core Model' when the overall process rate is limited by both intergrain diffusion and the chemical reaction. By analogy with the 'Shrinking Unreacted Core Model', the modification consisted of introducing an $X$ (fractional conversion degree) in the numerator of the second term of the denominator of the second member of that equation. This yielded: 


$$
\frac{d X}{d t}=\left(\frac{1}{c_{B}^{0}}\right) \cdot\left[\frac{K_{C}-b \cdot c_{Q}^{G}}{\frac{K_{C}}{k \cdot S_{s p}(1-X)^{1 / 3}}+\frac{r_{S}^{2} \cdot X}{3 \cdot D_{e}}}\right]
$$

This modification was successful as shown below.

\subsection{Experiments conducted in air atmosphere}

These experiments enabled the suitability of equation (5) to fit to the experimental results obtained in air atmosphere to be verified, and the values of the kinetic parameters $k$ and $D_{e}$ corresponding to the different test temperatures to be determined.

When the calcite decomposition experiments were conducted in air atmosphere, $c^{G}{ }_{Q}=0$ in equation (5), yielding:

$$
\frac{d X}{d t}=\left(\frac{1}{c_{B}^{0}}\right) \cdot\left[\frac{K_{C}}{\frac{K_{C}}{k \cdot S_{s p}(1-X)^{1 / 3}}+\frac{r_{S}^{2} \cdot X}{3 \cdot D_{e}}}\right]
$$

For the calcite particles with an average radius equal to or smaller than $0.225 \mathrm{~mm}$, the second term in the denominator of the second member of that equation was negligible compared to the first term, as shown elsewhere [40], so that in this particle size range, the above equation reduces to:

$$
\frac{d X}{d t}=\left(\frac{1}{c_{B}^{0}}\right) \cdot k \cdot S_{s p} \cdot(1-X)^{1 / 3}
$$

It was possible to integrate equations (7) and (6) analytically, starting with the boundary conditions $X=0 ; t=t_{0}$, respectively yielding:

$$
\begin{aligned}
& t=t_{0}+\frac{3 \cdot c_{B}^{0}}{2 \cdot k \cdot S_{s p}}\left(1-(1-X)^{2 / 3}\right) \\
& t=t_{0}+\frac{3 \cdot c_{B}^{0}}{2 \cdot k \cdot S_{s p}}\left(1-(1-X)^{2 / 3}\right)+\frac{r_{s}^{2} \cdot c_{B}^{0} \cdot X^{2}}{6 \cdot D_{e} \cdot K_{c}}
\end{aligned}
$$


Since the samples are introduced into the reactor at a temperature lower than the preselected to develop the experiment (see section 2.3), $t_{0}$ is considered as the time required for the sample to reach the preselected temperature, in every experiment. Hereinafter, $t_{0}$ will be called "induction time".

Equation (8) was applied to the experimental results obtained with the particles that had an average radius equal to $0.225 \mathrm{~mm}$, using different pairs of values of the kinetic constant $(k)$ and induction time $\left(t_{0}\right)$, until the curve that best fitted to the experimental results was obtained, by trial and error, for each test temperature. The selected values of $k$ for each test temperature are presented in Table 1.

Table 1

The values of $K_{c}$ were calculated, at each temperature, from the equation:

$$
K_{c}=\frac{P_{Q}^{0}}{R \cdot T}
$$

where $P_{Q}^{0}$ (thermal dissociation pressure of calcium carbonate at temperature $\mathrm{T}$, in $\mathrm{kPa}$ ) was calculated from the Hill equation [42]:

$$
P_{Q}^{0}=(0.1333) \cdot\left(10^{10.4022-8792.3 / T}\right)
$$

The $P^{0}{ }_{Q}$ values obtained using equation (11), in the studied range of temperatures, practically coincided with those calculated using the equation proposed by Barin [36, 43].

Using the values of $k$ and $K_{c}$ obtained at each temperature, equation (9) was fitted by trial and error to the experimental results corresponding to the fractions of average radius larger than $0.225 \mathrm{~mm}$, using different values of $D_{e}$ and $t_{0}$, until the best fit was obtained. The $K_{c}$ values calculated from equations (10) and (11) and the values of $D_{e}$ obtained from the best fit, in each series of experiments, are shown in Table 1.

In all the experiments, the values obtained for $\mathrm{t}_{0}$ were comprised in the range 0.1-0.9 minutes. At each studied temperature, the $t_{0}$ value increases as the particle radius is increased. At each value of particle radius, $t_{0}$ slightly decreased when increased the operating temperature.

The variation of the values of $k$ with temperature was fitted to the following equation: 


$$
k=22.5 \cdot \exp \left(-\frac{139000}{8.314 \cdot T}\right)
$$

with a regression coefficient of 0.9994 .

The value obtained for the apparent activation energy $(139000 \mathrm{~J} / \mathrm{mol})$ is of the same order as or intermediate among the values proposed by other researchers $[14,44-49]$ that have studied this process (Table 2).

\section{Table 2}

Substituting equations (10) and (12) in equation (9) and using, at each test temperature, the experimentally determined corresponding values of $D_{e}$ and $t_{0}$, several pairs of values of $X=$ $f(t)$ were calculated for every studied particle size fraction. The values obtained have been plotted, in the form $X=f(t)$, as solid lines in Figures 4 to 8 together with the experimental results.

The calculated values for $X$ fitted very well to the experimental data, in the entire studied temperature range (1123-1223 K), for particle size fractions with an average radius smaller than $1.025 \mathrm{~mm}$.

For particles with an average radius equal to or larger than $1.02 \mathrm{~mm}$, equation (9) only fitted well to the experimental data in the conversion range of $0-0.95$. This behaviour may be due to the fact that the influence of diffusion increases with particle size, so that this step could become the limiting step of the process in the higher range of the conversion degree.

It may be concluded, therefore, that equation (5) enables the results of the thermal decomposition process of the studied calcite particles to be predicted, when the process is conducted in air atmosphere in the studied range of operating conditions.

When equations (5) or (9) were applied to the results obtained in the previous paper [40] with calcite particles of average radius smaller than $0.225 \mathrm{~mm}$, they also fitted very well to the data. This is because in this range of sizes of $r_{s}$, as indicated above, the value of the second term of the denominator in the second member of equation (5) is negligible compared to the value of the first term, so that the value of the particle radius hardly influences the relationship between $X$ and $t$. 
With regard to the value of the effective diffusivity $(D e)$ obtained (Table 1), it may be noted that this decreased slightly from $3.4 \cdot 10^{-4} \mathrm{~m}^{2} \bullet$ minute ${ }^{-1}$ to $3.2 \cdot 10^{-4} \mathrm{~m}^{2} \bullet$ minute ${ }^{-1}$ when the temperature increases from 1123 to $1148 \mathrm{~K}$ and, after, also decreases from $3.25 \bullet 10^{-4}$ $\mathrm{m}^{2} \cdot$ minute $^{-1}$ to $2.85 \cdot 10^{-4} \mathrm{~m}^{2} \bullet$ minute $^{-1}$ when the temperature increases from 1173 to $1198 \mathrm{~K}$.

This atypical behaviour might be caused by a change in the structure of the calcite particles [21], produced at these temperatures, owing to an incipient sintering process, which could reduce their porosity. Bear in mind that the effective diffusivity in porous solids is in direct proportion with their porosity [13, 48-52].

In order to try to confirm this hypothesis, the porosimetry of samples of the calcite particle fraction with an average radius of $0.70 \mathrm{~mm}$, subsequently to be treated in the reactor for 15 minutes at different temperatures, was determined. Table 3 shows the values obtained for the total pore volume and apparent porosity of the thermally treated samples. It may be observed that, at temperatures above $1123 \mathrm{~K}$, the value of both properties decreased slightly, which could explain the results obtained. The values obtained for porosity in these experiments are of the same order as the porosities reported by other researchers for certain types of calcined limestone [36,52].

\section{Table 3}

The values of $D_{e}$ obtained are comparable to those proposed by Rajeswara Rao [53] for pellets, obtained by compacting $\mathrm{CaCO}_{3}$ powder, treated at different temperatures $\left(1.44 \cdot 10^{-4}\right.$ $\mathrm{m}^{2} \cdot \mathrm{min}^{-1}$ and $1.3 \cdot 10^{-4} \mathrm{~m}^{2} \cdot \mathrm{min}^{-1}$ for pellets treated at $1073 \mathrm{~K}$ and $973 \mathrm{~K}$ respectively) and for pellets obtained by compacting powdered samples of the product resulting from the thermal decomposition of $\mathrm{CaCO}_{3}$ pellets at different temperatures $\left(1.5 \cdot 10^{-4}, 1.21 \cdot 10^{-4}\right.$, and $1.14 \cdot 10^{-4}$ $\mathrm{m}^{2} \cdot \mathrm{min}^{-1}$ for pellet decomposition at 1103,1053 , and $973 \mathrm{~K}$, respectively).

The diffusivity values proposed by Campbell and Hills [50] ranged from $5.64 \cdot 10^{-4}$ to $16.8 \cdot 10^{-}$ ${ }^{4} \mathrm{~m}^{2} \cdot \mathrm{min}^{-1}$ for porosities of 0.47 and 0.7 , respectively.

More information about $D_{e}$ has been added in section 4.4.2 as a consequence of the results obtained in a set of additional experiments. 


\subsection{Experiments conducted in the presence of carbon dioxide. Influence of the} $\mathrm{CO}_{2}$ concentration in the gas stream

When the experiments were conducted only in $\mathrm{CO}_{2}$ or in mixtures of air and carbon dioxide, it was possible to integrate equation (5) analytically, starting also with the boundary conditions $X=0 ; t=t_{0}$, which yielded:

$$
t=t_{0}+\frac{3 \cdot c_{B}^{0} \cdot K_{c}}{2 \cdot k \cdot S_{s p}\left(K_{c}-b \cdot c_{Q}^{G}\right)}\left(1-(1-X)^{2 / 3}\right)+\frac{r_{s}^{2} \cdot c_{B}^{0} \cdot X^{2}}{6 \cdot D_{e}\left(K_{c}-b \cdot c_{Q}^{G}\right)}
$$

In these series of experiments, the $\mathrm{CO}_{2}$ molar concentration in the gaseous phase $\left(c_{Q}^{G}\right)$ was calculated from the equation:

$$
c_{Q}^{G}=\frac{P \cdot y_{Q}^{G}}{R \cdot T}
$$

where $P$ and $T$ are the pressure $(\mathrm{kPa})$ and temperature $(\mathrm{K})$ in the reactor and $y^{G}{ }_{Q}$ is the $\mathrm{CO}_{2}$ molar fraction in the gaseous phase in each series of experiments. The pressure in the reactor ranged from $99-101 \mathrm{kPa}$ in all the experiments.

\subsubsection{Experiments conducted in $\mathrm{CO}_{2}$ atmosphere}

The possibility was studied, first, of correlating the results obtained in the more extreme conditions, i.e. those obtained in the experiments conducted in Figure 9 with equation (13).

For this, at each studied temperature, using the values of $k$ and $K_{c}$ calculated from eq. (12) and eqs. (10) and (11) and the values of $D_{e}$ obtained by interpolation from the determined values in section 4.2 (Table 1), different values of the induction time $\left(t_{0}\right)$ and the parameter $b$ were tested by trial and error until the best fit was achieved.

The values of $k, K_{c}$, and $D_{e}$ used, as well as those of parameter $b$ obtained at each test temperature and $\mathrm{CO}_{2}$ concentration in gas phase, calculated from equation (14) doing $y_{Q}^{G}=1$, are detailed in Table 4.

\section{Table 4}


The pairs of values of $X=f(t)$ calculated for each test temperature, corresponding to the $b$ and $t_{0}$ values that best fitted to the experimental results, have been plotted in Figure 9 as solid lines, together with the experimental results. In all the experiments, the values obtained for $t_{0}$ were lower than 0.4 minutes. The calculated values fitted very well to the experimental data, in all the studied temperature range: 1150 to $1216 \mathrm{~K}$.

The fact that it has been necessary to use values of parameter $b$ different from unity to fit the experimental results to equation (13) seems to confirm that the $\mathrm{CO}_{2}$ concentrations on both sides of the gas-calcite particle interface $\left(c_{Q S}^{G}\right.$ and $c_{Q S}^{S}$ ) during the calcite particle thermal decomposition process are different, and their relationship could be subject to an equilibrium law as it had been foreseen, in principle, when the proposed equations were deduced (see equation A.4 in the Appendix). This circumstance could be due to the fact that in the studied decomposition process, according to some researchers [2,15,35,36,37], a (physical or chemical) $\mathrm{CO}_{2}$ adsorption phenomenon could develop at the particle surface, whose equilibrium constant would be related to parameter $b$ through an expression analogous to equation A.4.

The examination of Figure 9 suggests that the calcite particle decomposition process appears to be practically inhibited at temperatures below $1150 \mathrm{~K}$, when it conducts in pure $\mathrm{CO}_{2}$ atmosphere, because it develops very slow at this temperature, as the corresponding curve $X$ $=f(t)$ shows.

In order to attempt to confirm these results, the decomposition was studied of the calcite particle size fractions with an average radius of $0.225 \mathrm{~mm}$ and $0.70 \mathrm{~mm}$, using the DTA-TG technique, in a stream of $\mathrm{CO}_{2}$. The two tested particle sizes yielded the same result. The $T G$ $D T G$ thermograms obtained using the fraction with an average radius of $0.70 \mathrm{~mm}$, operating in a dynamic atmosphere of $\mathrm{CO}_{2}$ at an absolute pressure of $100.7 \mathrm{kPa}$ and a heating rate of 10 ${ }^{\circ} \mathrm{C} / \mathrm{min}$, are shown in Figure 15.

\section{Figure 15}

As may be observed, calcite particle decomposition seems begin at 1148-1149 K, a temperature that is practically of the same order as the operating temperature at which the 
calcite particles start to decompose $(1150 \mathrm{~K})$ in the series of experiments conducted in $\mathrm{CO}_{2}$ atmosphere, in the reactor (Figure 9).

This result confirms the validity of the experimental results obtained in the reactor.

\subsubsection{Experiments conducted in different concentrations of air and $\mathrm{CO}_{2}$}

In order to verify the effectiveness of equation (13) for correlating the experimental results obtained in the calcite thermal decomposition tests when they were carried out in different concentrations of air and $\mathrm{CO}_{2}$, the values of the physical properties of the particles used (average radius $=0.70 \mathrm{~mm})$, as well as the values of the rate parameters $\left(k, K_{c}, D_{e}\right.$ and $\left.b\right)$ obtained for the different operating temperatures tested (table 4) and the corresponding values of $c_{Q}{ }^{G}$ calculated from equation (14), were substituted in that equation.

Different values were tested, by trial and error, for induction time $\left(t_{0}\right)$ and the parameter $b$ at each studied temperature, starting on the $t_{0}$ values obtained in the corresponding experiments conducted in air atmosphere until the best fit was obtained The value of constant $b$ obtained at each experiment and the test temperature and $\mathrm{CO}_{2}$ concentration in the gas phase corresponding are detailed also in Table 4.

The values of $X=f(t)$ calculated using the values of $t_{0}$ that best fitted to the experimental results, corresponding to the temperatures 1123, 1152,1173, 1195 and $1216 \mathrm{~K}$, are plotted (as solid lines) together with the experimental data in Figures 10 to 14. As can see, the agreement between the experimental data and the calculated values is very good.

As it has indicated above (Section 3.2.2), the values of $X=f(t)$ calculated for the experiments conducted at the temperatures $1113\left(53 \%\right.$ of $\left.\mathrm{CO}_{2}\right), 1107\left(53 \%\right.$ of $\left.\mathrm{CO}_{2}\right)$ and $1098(38 \%$ de $\mathrm{CO}_{2}$ ) have not been represented versus the corresponding experimental results to reduce the number of figures in this paper. These experiments were developed to enlarge the information about the behaviour of effective diffusivity $\left(D_{e}\right)$ and the parameter $b$, with the changes of operating temperature and the $\mathrm{CO}_{2}$ concentration in the gaseous phase. The values of the corresponding kinetic constants, that better fit the experimental data, on applying equation (13), are shown in Table 4. In this case, the agreement among the calculated values and the experimental was also very good. 
These results demonstrate the validity of equations (5) or (13) for correlating the experimental data and predicting the relation $X=f(t, T)$ in the studied range of operating conditions. They confirm, moreover, that the reverse chemical reaction ( $\mathrm{CaO}$ carbonation) of reaction scheme (1) is first order with respect to carbon dioxide, in all the studied temperature range (1123$1216 \mathrm{~K})$, unlike the results obtained by Rao [18].

4.4. Considerations on the values obtained for effective diffusivity $\left(D_{e}\right)$ and for parameter (b) of equation (A.4)

\subsubsection{Considerations on the effective diffusivity $\left(D_{e}\right)$}

To complete the information that has been provided in section 4.1, about from the influence of operating temperature on the value of the effective diffusivity of $\mathrm{CO}_{2}$ through the particle, additional four series of experiments, in air atmosphere, at four temperatures $(975,1028,1047$ and $1080 \mathrm{~K}$ ) below the minimum tested $1098 \mathrm{~K}$, have been carried out. The values of the kinetic constants that best fit the experimental data, on applying equations (8) and (9) are also shown in Table 4.

In Figure 16 have been plotted, in semilogarithmic coordinates, the values of $D_{e}$, that are shown in Tables 1 and 4, versus the inverse of the corresponding operating temperature. As can see, in the temperature ranges in which there are not changes in the porous structure of the particles, the effective diffusivity increases very slightly with temperature, in accordance with an exponential relationship.

The two slumps in the diffusivity that occur around $1123 \mathrm{~K}$ and $1173 \mathrm{~K}$, are probably driven by decreases in porosity of the solid, that it has already been commented in Section 4.1.

\section{Figure 16}

\subsubsection{Considerations on the parameter $b$}

The values of parameter $b$, detailed in Table 4 , have been plotted in the form $\ln (b)$ versus $1 / T$ in Figure 17. As may be observed, these values were aligned in four straight lines with 
negative slope, one for each $\mathrm{CO}_{2}$ concentration in the studied gas phase. All these results have been correlated with a single equation of the form:

$$
b=\left(1.096+0.190 \cdot y_{Q}^{G}\right) \cdot \exp \left[\frac{-602.3}{T}\right]
$$

With this equation it is possible to predict, quite accurately, the value of parameter b starting from the values of the operating temperature and of the $\mathrm{CO}_{2}$ molar fraction of in the gaseous phase.

\section{Figure 17}

In support of the assumption represented by equation A.4, it may be noted that in the DTATG tests, conducted in a stream of $\mathrm{CO}_{2}$ at an absolute pressure of $100.7 \mathrm{kPa}$, calcite particle decomposition began at 1148-1149 K (figure 15). This temperature is of the order of $20 \mathrm{~K}$ lower than that predicted by the respective law of chemical equilibrium. Indeed, according to the equations proposed in different studies on the variation of the dissociation pressure of calcium carbonate with temperature, at a dissociation pressure of $100.7 \mathrm{kPa}$ (equal to the $\mathrm{CO}_{2}$ partial pressure used in the TG tests) the calcite particles should begin to decompose at the following temperatures: $1169 \mathrm{~K}$ (Barin) [43]; $1170 \mathrm{~K}$ (Hill and Winter [42] and Johnston [55]); 1172 K (Southard and Royster [56] and Kelley and Anderson [57]).

The difference existing between the value calculated for the dissociation temperature of the $\mathrm{CaCO}_{3}$ when applying the law of chemical equilibrium and the decomposition temperature determined by the DTA-TG experiments might be explained by assuming that, when the calcite particle thermal decomposition process takes place in $\mathrm{CO}_{2}$ atmosphere, there is a cause, other than the law of chemical equilibrium, which influences the temperature at which this decomposition occurs. This cause, as suggested above, could be a phenomenon of physical adsorption of $\mathrm{CO}_{2}$ on the $\mathrm{CaO}$ resulting from the calcite decomposition.

This adsorption phenomenon would be responsible for the difference between the carbon dioxide concentrations on both sides of the gas-calcite interface during the process studied, whose relationship would be governed by some physical equilibrium law, as it had been foreseen in equation (A.4) used to derive equation (14).. 


\section{Conclusions}

The calcium carbonate thermal decomposition process in calcite particles $(0.45-3.60 \mathrm{~mm}$ of average diameter), made up of aggregates of microcrystals smaller than $0.020 \mathrm{~mm}$, was studied in the temperature range $975-1216 \mathrm{~K}$. The experiments were carried out in air atmosphere, in $\mathrm{CO}_{2}$ atmosphere, and also in a gas stream comprising different concentrations of air and $\mathrm{CO}_{2}$.

A single equation, based on the 'Grainy Pellet Model', is proposed in this study, assuming that both the chemical reaction in the microcrystals and $\mathrm{CO}_{2}$ diffusion through the particle structure are process-rate limiting and that the reverse reaction in scheme (1) is a first-order reaction with regard to the $\mathrm{CO}_{2}$ concentration at the reaction interface. It is also assumed that the relationship between the $\mathrm{CO}_{2}$ concentrations on both sides of the solid-gas interface seems to be governed by an equilibrium law that could derive from a physical adsorption phenomenon of the carbon dioxide on the $\mathrm{CaO}$ formed as reaction product in each particle.

This proposed equation relates the calcium carbonate conversion degree in the calcite particles to the reaction time and operating conditions (temperature and $\mathrm{CO}_{2}$ concentration in the gas phase, as well as the specific surface area, initial calcium carbonate concentration, and average particle radius).

This equation satisfactorily fits to the experimental results obtained in the entire studied range of operating conditions, working in air, in $\mathrm{CO}_{2}$, and also in different mixtures of air and $\mathrm{CO}_{2}$ in the entire studied temperature range (975-1216 K).

The values obtained for the apparent activation energy of the chemical reaction step and for the $\mathrm{CO}_{2}$ effective diffusivity through the particle during the thermal decomposition process, using this equation, are of the same order as those proposed by other researchers.

\section{Appendix}

\section{Deduction of equation (4)}


Using generalised nomenclature, the scheme of chemical reaction (1) may be expressed as:

$$
-v_{B} B(s)=v_{P} P(s)+v_{Q} Q(g)
$$

where $\quad B=\mathrm{CaCO}_{3}, \quad P=\mathrm{CaO}, Q=\mathrm{CO}_{2}$, and $-v_{B}=v_{P}=v_{Q}=1$

Figure A.1 shows a scheme of the proposed kinetic model. The meanings of the symbols used in this figure and those that will be used in the rest of this section are explained in the Nomenclature.

\section{Figure A.1}

The kinetic model chosen to interpret the experimental results is based on the Grainy Pellet Model, given the grainy structure of the studied particles (Figures 1 and 2) and in order to be consistent with the model proposed for the same type of particles in a previous paper [40].

The selected kinetic model is based on the assumptions set out in that paper, on the assumptions made in section 4.1 of this paper, and on those detailed below:

a) Since the individual particles studied were very small (average radius $\leq 1.80 \mathrm{~mm}$ ), it is assumed that the internal temperature gradient in every calcite particle is negligible during the decomposition process that unfolds under isothermal conditions.

b) Each particle is assumed to behave as a homogeneous porous solid whose volume does not change during the decomposition process.

c) In each studied particle size fraction, all the particles are assumed to have the same size, which is equal to the average size of the corresponding granulometric size fraction.

d) The $\mathrm{CO}_{2}$ intragrain diffusion step (inside each $\mathrm{CaCO}_{3}$ microcrystal) is assumed not to influence the overall process rate, because it develops more quickly than the chemical reaction and intergrain diffusion steps, given the very small size of the microcrystals.

e) Equation (2) with exponent $n=1$ is used for the chemical reaction rate. The reaction interface is assumed to be the $\mathrm{CaCO}_{3} / \mathrm{CaO}$ boundary in every $\mathrm{CaCO}_{3}$ microcrystal making up 
the particle. In a first approximation, the reaction interface is assumed to be equal to the overall available inner and outer particle area $\left(S_{i}\right)$, which it is assumed may be calculated from the expression $[14,29,46]$ :

$$
S_{i}=S_{s p} \cdot V_{p B}^{0} \cdot(1-X)^{m} \quad\left(\mathrm{~m}^{2}\right)
$$

where the value of exponent $m$ depends on the shape and characteristics of the particle.

Consequently, the chemical reaction rate of the calcium carbonate decomposition in each calcite particle may be expressed by the equation:

$$
R_{B}=v_{B} \cdot S_{s p}^{0} \cdot V_{p B}^{0} \cdot(1-X)^{m} \cdot k \cdot\left(1-c_{Q i}^{S} / K_{C}\right) \quad\left(\operatorname{mol~B} \cdot \min ^{-1}\right)
$$

f) The concentration gradient responsible for $\mathrm{CO}_{2}$ diffusion through the particle pores is assumed to remain practically constant throughout the process and to be equal to the difference $\left(c^{s}{ }_{Q i}-c^{s}{ }_{Q S}\right)$, where $c^{s}{ }_{Q i}$ is assumed to be equal to the $\mathrm{CO}_{2}$ concentration calculated from the dissociation pressure of calcium carbonate at the system temperature.

g) The $\mathrm{CO}_{2}$ transfer step from the outer surface of the particle to the gaseous phase is assumed that takes place instantaneously and the relationship between the $\mathrm{CO}_{2}$ concentrations on both sides of this interface, $c_{Q S}^{G}$ and $c_{Q S}^{S}$ (see their meaning in Nomenclature and in Figure A.1), is of the form:

$$
c_{Q_{S}}^{S}=b \cdot c_{Q_{S}}^{G}
$$

where $b$ could be a representative constant of an equilibrium law at that interface. This equilibrium relationship could derive from a physical adsorption phenomenon of carbon dioxide on the $\mathrm{CaO}$ formed as reaction product in each particle.

h) The influence of the $\mathrm{CO}_{2}$ transfer step from the particle surface to the gas stream circulating around the particles, when the experiments are conducted in the presence of air or air and $\mathrm{CO}_{2}$ mixtures, is assumed to be negligible because a sufficiently high gas flow rate is used. This was experimentally verified.

Most of these assumptions are approximate and have been made to simplify the kinetic model in order to obtain relatively simple equations or to improve the fit of the resulting equations to the experimental data. 
In order to obtain a differential equation that related calcium carbonate conversion $(X)$ in the calcite particles to reaction time $(t)$, the mass balance in a non-steady state was applied to a particle, considering the reactant $B\left(\mathrm{CaCO}_{3}\right)$ as the reference component. This yielded:

$$
R_{B}=\frac{d N_{B}}{d t}=-N_{B}^{0} \cdot \frac{d X}{d t}=-V_{p B}^{0} \cdot c_{B}^{0} \cdot \frac{d X}{d t} \quad\left(\mathrm{kmol} \mathrm{B} \cdot \mathrm{min}^{-1}\right)
$$

In accordance with reaction scheme (A.1), the calcium carbonate decomposition rate $\left(R_{B}\right)$ and the $\mathrm{CO}_{2}$ formation rate $\left(R_{Q}\right)$ are related by the expression:

$$
R_{B}=R_{Q} \cdot\left(\frac{v_{B}}{v_{Q}}\right)
$$

Since all the $\mathrm{CO}_{2}$ given off by the decomposition reaction needs to move to the outside of the particle, assuming a quasi-steady-state regime during the process, the following must hold:

$$
R_{\mathrm{Q}}=\mathrm{W}_{\mathrm{Q}}^{\mathrm{S}}=\mathrm{W}_{\mathrm{Q}}^{\mathrm{G}}
$$

Eqs. (A.6) and (A.7), taking into account the values of the stoichiometric coefficients of the reaction scheme represented by equation (A.1), yield:

$$
-R_{B}=R_{Q}=W_{Q}^{S}=W_{Q}^{G}
$$

In order to select a rate equation for the $\mathrm{CO}_{2}$ diffusion step through the particle, several possibilities were tested. The rate equation that led to the final expression of $X=f(t)$ that best fitted the experimental results was the following:

$$
W_{Q}^{S}=S_{e x}^{0} \cdot \frac{D_{e}}{r_{S}} \cdot\left(c_{Q i}^{S}-c_{Q S}^{S}\right)
$$

where $S_{e x}^{0}$ is the particle outer surface. Equation (A.9) represents the diffusion, in a steady state, through a flat slab of parallel faces. This means assuming that calcite particles have an approximately cubic shape, which can be considered acceptable in view of Figure 1.

If it is assumed that that the turbulent transfer step of $\mathrm{CO}_{2}$ from the particle surface to the gas stream is very rapid compared to the rate of the two steps mentioned previously, it may be 
assumed that $c^{G}{ }_{Q S} \approx c^{G}{ }_{Q}$ and if it is also assumed that $c^{S}{ }_{Q S}=b \cdot c^{G}{ }_{Q S}$ is obeyed at the gas-particle interface, the equation (A.9) may be rewritten in the form:

$$
W_{Q}^{S}=S_{e x}^{0} \cdot \frac{D_{e}}{R_{S}} \cdot\left(c_{Q i}^{S}-b \cdot c_{Q}^{G}\right)
$$

The assumption $c_{Q S}^{G}=c_{Q}^{G}$ is particularly true when the only gas phase in contact with the calcite particles is $\mathrm{CO}_{2}$.

From the first and the third members of equation (A.8), taking into account equations (A.3) and (A.10), one obtains:

$$
-R_{B}=\frac{K_{C}-c_{Q i}^{S}}{\frac{K_{C}}{k \cdot S_{s p} \cdot V_{p B}^{0} \cdot(1-X)^{m}}}=\frac{c_{Q i}^{S}-b \cdot c_{Q}^{G}}{\frac{r_{S}}{S_{e x}^{0} \cdot D_{e}}}=\frac{K_{C}-b \cdot c_{Q}^{G}}{\frac{K_{C}}{k \cdot S_{s p} \cdot V_{p B}^{0} \cdot(1-X)^{m}}+\frac{r_{S}}{S_{e x}^{0} \cdot D_{e}}}
$$

(A.5) and (A.11) then yield:

$$
\frac{d X}{d t}=\frac{1}{c_{B}^{0}} \cdot \frac{K_{C}-b \cdot c_{Q}^{G}}{\frac{K_{C} \cdot V_{p B}^{0}}{k \cdot S_{s p} \cdot V_{p B}^{0} \cdot(1-X)^{m}}+\frac{r_{S} \cdot V_{p B}^{0}}{S_{e x}^{0} \cdot D_{e}}}
$$

and taking into account the definition of 'Sauter mean diameter' [39]:

$$
\frac{V_{p B}^{0}}{S_{e x}^{0}}=\frac{(4 / 3) \cdot \pi \cdot r_{S}^{3}}{4 \cdot \pi \cdot r_{S}^{2}}=\frac{r_{S}}{3}
$$

Finally, (A.12) and (A.13) yield:

$$
\frac{d X}{d t}=\frac{1}{c_{B}^{0}} \cdot \frac{K_{C}-b \cdot c_{Q}^{G}}{\frac{K_{C}}{k \cdot S_{s p} \cdot(1-X)^{m}}+\frac{r_{S}^{2}}{3 \cdot D_{e}}}
$$

\section{Nomenclature}

Symbol Name (Units)

$b \quad$ equilibrium constant in equation (A.4)

$c_{B}^{0} \quad$ initial molar concentration of $\mathrm{CaCO}_{3}$ in the particle $\left(\mathrm{kmol} \cdot \mathrm{m}^{-3}\right)$

$c_{Q}^{G} \quad$ molar concentration of $\mathrm{CO}_{2}$ in the gas phase $\left(\mathrm{kmol} \cdot \mathrm{m}^{-3}\right)$

$c_{Q S}^{G}$ molar concentration of $\mathrm{CO}_{2}$ in the gas phase, at the particle-gas interface $\left(\mathrm{kmol} \cdot \mathrm{m}^{-3}\right)$

$c_{Q S}^{S}$ molar concentration of $\mathrm{CO}_{2}$ in the particle pores at the particle-gas interface $\left(\mathrm{kmol} \cdot \mathrm{m}^{-3}\right)$

$c_{Q i}^{S}$ molar concentration of $\mathrm{CO}_{2}$ (in the particle pores) at the chemical reaction interface 
$\left(\mathrm{kmol} \cdot \mathrm{m}^{-3}\right)$

$c_{Q e}^{S} \quad \mathrm{CO}_{2}$ concentration in equilibrium with the solid at the pellet temperature $\left(\mathrm{kmol} \cdot \mathrm{m}^{-3}\right)$

$D_{e} \quad$ effective diffusion coefficient of $\mathrm{CO}_{2}$ through the particle $\left(\mathrm{m}^{2} \cdot\right.$ minute $\left.{ }^{-1}\right)$

$k \quad$ rate constant of the direct reaction in equation $(1)\left(\mathrm{kmol} \cdot \mathrm{m}^{-2} \cdot\right.$ minute $\left.^{-1}\right)$

$K_{c}$ equilibrium constant of reaction $(1)\left(\mathrm{kmol} \cdot \mathrm{m}^{-3}\right)$

$n \quad$ reaction order in equation (2)

$N^{0}{ }_{B}$ initial moles of $\mathrm{CaCO}_{3}$ in a particle $(\mathrm{kmol})$

$N_{B}$ moles of $\mathrm{CaCO}_{3}$ in a particle after time $t$ has elapsed $(\mathrm{kmol})$

$P \quad$ pressure in the reactor $(\mathrm{kPa})$

$p_{Q S}^{G} \mathrm{CO}_{2}$ partial pressure at the particle-gas interface, gas side $(\mathrm{kPa})$

$P_{Q}^{0} \quad$ dissociation pressure of calcium carbonate at temperature $\mathrm{T}(\mathrm{kPa})$

$r$ decomposition reaction rate in equation $(1)\left(\mathrm{kmol} \cdot \mathrm{m}^{-2} \cdot\right.$ minute $\left.^{-1}\right)$

$R \quad$ universal gas constant $\left(8.317 \mathrm{kPa} \cdot \mathrm{m}^{3} \cdot \mathrm{kmol}^{-1} \cdot \mathrm{K}^{-1}\right)$

$r_{S} \quad$ initial radius of the sphere with the same surface as the particle $(\mathrm{m})$.

$R_{j} \quad$ decomposition reaction rate in reference to $\mathrm{A}_{\mathrm{j}}\left(\mathrm{kmol} \mathrm{A}_{\mathrm{j}} /\right.$ minute $) ;\left(\mathrm{A}_{\mathrm{j}}=\mathrm{B}, \mathrm{Q}, \mathrm{P}\right)$

$S_{i} \quad$ reaction interface area $\left(\mathrm{m}^{2}\right)$

$S_{e x}^{0} \quad$ solid-gas interface area (particle outer surface) $\left(\mathrm{m}^{2}\right)$

$S_{s p} \quad$ specific surface area of the $\mathrm{CaCO}_{3}$ particle $\left(\mathrm{m}^{2} / \mathrm{m}^{3}\right.$ of the particle)

$t \quad$ residence time (minute)

$t_{0} \quad$ induction time (minute)

$T$ temperature $(\mathrm{K})$

$V_{p B}^{0}$ initial volume of the $\mathrm{CaCO}_{3}$ particle $\left(\mathrm{m}^{3}\right)$

$W_{Q}^{S} \quad \mathrm{CO}_{2}$ flow rate by diffusion from the reaction interface to the solid-gas interface $(\mathrm{kmol}$ $\mathrm{CO}_{2} \cdot$ minute $\left.^{-1}\right)$

$W_{Q}^{G} \mathrm{CO}_{2}$ flow rate from the solid-gas interface to the gas phase $\left(\mathrm{kmol} \mathrm{CO}_{2} \cdot\right.$ minute $\left.^{-1}\right)$

$X \quad \mathrm{CaCO}_{3}$ degree of conversion

$y_{Q}^{G} \quad \mathrm{CO}_{2}$ molar fraction in the gas phase

$\Delta M$ mass loss of the sample at a given residence time

$\Delta M_{*}$ mass loss of the sample at a sufficiently long residence time to achieve constant weight

Greek letters

$v_{B}, v_{Q}, v_{P}$ stoichiometric coefficients of $\mathrm{CaCO}_{3}, \mathrm{CO}_{2}$, and $\mathrm{CaO}$ in reaction scheme (A.1) 


\section{References}

[1] L. Caixin, Z. Lei, D. Jiguang, M. Qing, D. Hongxing, H. Hong, Surfactant-Aided Hydrothermal Synthesis and Carbon Dioxide Adsorption Behavior of ThreeDimensionally Mesoporous Calcium Oxide Single-Crystallites with Tri-, Tetra-, and Hexagonal Morphologies. J. Phys. Chem. C 112 (2008) 19248-19256.

[2] E. Kadossov, U. Burghaus, Adsorption kinetics and dynamics of $\mathrm{CO}, \mathrm{NO}$, and $\mathrm{CO}_{2}$ on reduced CaO(100). J. Phys. Chem. C 112 (2008) 7390-7400.

[3] F. Voigts, F. Bebensee, S. Dahle, K. Volgmann, W. Maus-Friedrichs. The adsorption of $\mathrm{CO}_{2}$ and $\mathrm{CO}$ on $\mathrm{Ca}$ and $\mathrm{CaO}$ films studied with MIES, UPS and XPS. Surf. Sci. 603 (2009) 40-49.

[4] N.H. Florin, A.T. Harris. Reactivity of $\mathrm{CaO}$ derived from nano-sized $\mathrm{CaCO}_{3}$ particles through multiple $\mathrm{CO}_{2}$ capture-and-release cycles. Chem. Eng. Sci. 64 (2009) 187-191.

[5] S. Vivanpatarakij, N. Laosiripojana, W. Kiatkittipong, A. Arpornwichanop, A. Soottitantawat, S. Assabumrungrat. Simulation of solid oxide fuel cell systems integrated with sequential CaO- $\mathrm{CO}_{2}$ capture unit. Chem. Eng. J. 147 (2009) 336-341.

[6] H. Lu, P.G. Smirniotis, F.O. Ernst, S.E. Pratsinis. Nanostructured Ca-based sorbents with high $\mathrm{CO}_{2}$ uptake efficiency. Chem. Eng. Sci. 64 (2009) 1936-1943.

[7] M. Mofarahi, P. Roohi, F. Farshadpoor. Study of $\mathrm{CaO}$ Sorbent for $\mathrm{CO}_{2}$ Capture from Flue Gases. Chem. Eng. Trans. 17 (2009) 403-408.

[8] R. Inoue, S. Ueda, K. Wakuta, K. Sasaki, T. Ariyama. Thermodynamic Consideration on the Absorption Properties of Carbon Dioxide to Basic Oxide. Isij Int. 50 (2010) 1532-1538.

[9] J. Fernandez, F. Gonzalez, C. Pesquera, C. Blanco, M. J. Renedo. Study of the $\mathrm{CO}_{2} /$ Sorbent Interaction in Sorbents Prepared with Mesoporous Supports and Calcium Compounds. Ind. Eng. Chem. Res. 49 (2010) 2986-2991. 
[10] C.H. Huang, K.P. Chang, C.T. Yu, P.C. Chiang, C.F. Wang. Development of high-temperature $\mathrm{CO}_{2}$ sorbents made of CaO-based mesoporous silica. Chem. Eng. J. 161 (2010) 129-135.

[11] K. Wang, X. Guo, P.F. Zhao, C.G. Zheng. Cyclic $\mathrm{CO}_{2}$ capture of CaO-based sorbent in the presence of metakaolin and aluminum (hydr)oxides. Appl. Clay Sci. 50 (2010) 41-46.

[12] Y. Wang, S. Lin, Y. Suzuki. Experimental study on $\mathrm{CO}_{2}$ capture conditions of a fluidized bed limestone decomposition reactor. Fuel Process. Technol. 91 (2010) 958963.

[13] B.R. Stanmore, P. Gilot. Review-Calcination and carbonation of limestone during thermal cycling for $\mathrm{CO}_{2}$ sequestration. Fuel Process. Technol. 86 (2005) 1707-1743.

[14] S. Fujimoto, T. Hanaoka, H. Taniguchi, K. Kuramoto, Y. Matsumura, S.Y. Lin, T. Minowa. A Kinetic Study of the Decomposition of $\mathrm{CaCO}_{3}$ at $\mathrm{High} \mathrm{CO}_{2}$ Partial Pressure for the Regeneration of a $\mathrm{CO}_{2}$ Sorbent. J. Chem. Eng. Jpn 39 (2006) 1191-1194.

[15] A. I. Lysikov, A. N. Salanov, A. G. Okunev. Change of $\mathrm{CO}_{2}$ Carrying Capacity of $\mathrm{CaO}$ in Isothermal Recarbonation-Decomposition Cycles Ind. Eng. Chem. Res. 46 (2007) 4633-4638.

[16] Y. Wang, S. Lin, Y. Suzuki. Study of Limestone Calcination with $\mathrm{CO}_{2}$ Capture: Decomposition Behavior in a $\mathrm{CO}_{2}$ Atmosphere. Energ. Fuel. 21 (2007), 3317-3321.

[17] Y. Wang, S. Lin, Y. Suzuki. Limestone calcination with $\mathrm{CO}_{2}$ capture (II): Decomposition in $\mathrm{CO}_{2} /$ steam and $\mathrm{CO}_{2} / \mathrm{N}_{2}$ atmospheres. Energ. Fuel. 22 (2008) 23262331.

[18] T. Rajeswara Rao, D.J. Gunn, J.H. Bowen. Kinetics of calcium carbonate decomposition. Chem. Eng. Res. Des. 67 (1989) 38-47. 
[19] M.S. Murthy, B.R. Marish, K.S. Rajanandam, K.Y.A.P. Kumar. Investigation on the kinetics of thermal decomposition of calcium carbonate. Chem. Eng. Sci. 49 (1994) 2198-2204.

[20] J. Szekely, J.W. Evans. A structural model for gas-solid reactions with a moving boundary. Chem. Eng. Sci. 25 (1970) 1091-1107.

[21] J. Szekely, J.W. Evans. A structural model for gas-solid reactions with a moving boundary 2. Effect of grain size, porosity and temperature on reaction of porous pellets. Chem. Eng. Sci. 26 (1971) 1901-1913.

[22] H.Y. Sohn, J. Szekely. A structural model for gas-solid reactions with a moving boundary 3. General dimensionless representation of irreversible reaction between a porous solid and a reactant gas. Chem. Eng. Sci. 27 (1972) 763-778.

[23] R.L. Pigford, G. Sliger. Rate of diffusion-controlled reaction between a gas and a porous solid sphere. Ind. Eng. Chem. Proc. Des. Dev. 12 (1973) 85-91.

[24] H.Y. Sohn, J. Szekely. The effect of intragrain diffusion on the reaction between a porous solid and a gas. Chem. Eng. Sci. 29 (1974) 630-634.

[25] J. Szekely Gas-Solid Reactions, Academic Press, London, 1976, pp. 128-130.

[26] L.K. Doraiswamy, M.M. Sharma. Heterogeneous reactions: analysis, examples, and reactor design: volume 1: Gas-Solid and Solid-Solid Reactions, John Wiley and Sons, New York, 1984, pp. 458-460.

[27] M. Ishida, C.Y. Wen. Comparison of kinetic and diffusional models for solid-gas reactions. AIChE. J. 14 (1968) 311-317

[28] O. Levenspiel. The Chemical Reactor Omnibook, OSU Book Stores, Corvallis, 1979, pp. 55.4-55.7. 
[29] J. Khinast, G.F. Krammer, Ch. Brunner, G. Staundiger. Decomposition of limestone: the influence of $\mathrm{CO}_{2}$ and particle size on the reaction rate. Chem. Eng. Sci. 51 (1996) 623-634.

[30] L.K. Doraiswamy, M.M. Sharma. Heterogeneous reactions: analysis, examples, and reactor design: volume 1: Gas-Solid and Solid-Solid Reactions. John Wiley and Sons, New York, 1984, pp. 450-456.

[31] B.V. L'vov, L.K. Polzik, V.L. Ugolkov. Decomposition kinetics of calcite: a new approach to the old problem. Thermochim. Acta. 390 (2009) 5-19.

[32] H. Galai, M. Pijolat, F. Valdivieso, K. Nahdi, M. Trabelsi-Ayadi. Kinetic Study of the Second Stage of the Dolomite Thermal Decomposition. Ann. Chim-Sci. Mat. 33 (2008) 47-57.

[33] V. Bouineau, V. Pijolat, M. Soustelle. Characterisation of the chemical reactivity of a $\mathrm{CaCO}_{3}$ powder for its decomposition. J. Eur. Ceram. Soc. 18 (1998) 1319-1324.

[34] Y. Zheng, K. Song, B. Chi, C. Zeng. Decomposition Kinetics of $\mathrm{CaCO}_{3}$ in $\mathrm{CO}_{2}$ Atmosphere. J. Huazhong University of Science and Technology (Natural Science Edition) 35 (2007) 87-89.

[35] D. Berutto, R. Botter, A.W. Searcy. Thermodynamics and kinetics of carbon dioxide chemisorption on calcium oxide. J. Phys. Chem. 88 (1984) 4052-4055.

[36] F. Garcia-Labiano, A. Abad, L.F. de Diego, P. Gayan, J. Adanez. Calcination of calcium-based sorbents at pressure in a broad range of $\mathrm{CO}_{2}$ concentrations. Chem. Eng. Sci. 57 (2002) 2381-2393.

[37] J.S. Wang, E.J. Anthony. On the decay behavior of the $\mathrm{CO}_{2}$ absorption capacity of CaO-based sorbents. Ind. Eng. Chem. Res. 44 (2005) 627-629.

[38] O. Levenspiel. The Chemical Reactor Omnibook.; OSU Book Stores: Corvallis, 1979; pp (vii). 
[39] British standard BS 2955: 1993. Glossary of terms relating to particle technology. 1993.

[40] A. Escardino, J. Garcia-Ten, C. Feliu. Kinetic study of calcite particle (powder) thermal decomposition: Part I. J. Eur. Ceram. Soc. 28 (2008) 3011-3020.

[41] Calvelo, A.; Smith, J.M. In Proceedings of CHEMECA 70 1st Australian conference on Chemical Engineering. Butterworths: Chatswood, 1971.

[42] K.J. Hill, E.R.S. Winter. Thermal dissociation pressure of calcium carbonate. J. Phys. Chem. 60 (1956) 1361-1362.

[43] I. Barin. Thermochemical data of pure substances. Weinheim, VCH, 1989.

[44] T.R. Ingraham, P. Marier. Kinetic studies on the thermal decomposition of calcium carbonate. Can. J. Chem. Eng. 41 (1963) 170-173.

[45] A.W.D. Hills. The mechanism of the thermal decomposition of calcium carbonate. Chem. Eng. Sci. 23 (1968) 297-320.

[46] A.B. Fuertes, G. Marban, K. Rubiera. Kinetics of Thermal Decomposition of Limestone Particles in a Fluidized Bed Reactor. Chem. Eng. Res. Des. 71 (1993) 421428.

[47] T. Rajeswara Rao. Kinetics of calcium carbonate decomposition. Chem. Eng. Technol. 19 (1996) 373-377.

[48] K. Feng, S.J. Lombardo. Kinetic Analysis from Dilatometry and Mass Spectrometry Measurements of the Decomposition and Sintering of Calcium Carbonate. J. Ceram. Process. Res. 3 (2002) 101-8. 
[49] C. Rodriguez-Navarro, E. Ruiz-Agudo, A. Luque, A.B. Rodriguez-Navarro, M. Ortega-Huertas. Thermal Decomposition of Calcite: Mechanisms of Formation and Textural Evolution of $\mathrm{CaO}$ nanocrystals. Am. Miner. 94 (2009) 578-93.

[50] F.R. Campbell, A.W.D. Hills. Transport properties of porous lime and their influence on the decomposition of porous compacts of calcium carbonate. Chem. Eng. Sci. 25 (1970) 929-942.

[51] Beruto, D. T., R. Vecchiattini, and M. Giordani. Solid Products and Rate-Limiting Step in the Thermal Half Decomposition of natural Dolomite in a $\mathrm{CO}_{2}(\mathrm{~g})$ Atmosphere. Thermochim. Acta 405 (2003) 183-94.

[52] S.V. Krishnan, S.V. Sotirchos. Effective diffusivity changes during calcination, carbonation, recalcination, and sulfation of limestones. Chem. Eng. Sci. 48 (1994) 1195-1208.

[53] T. Rajeswara Rao. Effective diffusivities in calcium carbonate and calcium oxide solid pellets. Chem. Eng. Commun. 35 (1985) 203-209.

[54] J.M. Criado, M. Gonzalez, J. Malek, A. Ortega. The effect of the $\mathrm{CO}_{2}$ pressure on the thermal decomposition kinetics of calcium carbonate. Thermochim. Acta. 254 (1995) 121-127.

[55] J. Johnston. The thermal dissociation of calcium carbonate. J. Am. Chem. Soc. 32 (1910) 938-946.

[56] J.C. Southard, P.H. Royster. The thermal dissociation of calcium carbonate. J. Phys. Chem. 40 (1936) 435-438.

[57] K.K. Kelley and C.T. Anderson. Contributions to the data on theoretical metallurgy. IV. Metal carbonates-correlations and applications of thermodynamic properties. Bur. Mines Bull. 384 (1935) p. 25. 
Table 1. Values of $k, K_{c}$, and $D_{e}$ obtained in the best fits with Equations (8) and (9); $\left(c^{0}{ }_{B}\right.$ $=21.7 \mathrm{kmol} \cdot \mathrm{m}^{-3} ; S_{s p}=1354700 \mathrm{~m}^{2} \cdot \mathrm{m}^{-3}$ particle).

\begin{tabular}{|c|c|c|c|}
\hline $\mathrm{T}(\mathrm{K})$ & $\begin{array}{c}\mathrm{k} \cdot 10^{6} \\
\left(\mathrm{kmol} \cdot \mathrm{m}^{-2} \cdot \mathrm{min}^{-1}\right)\end{array}$ & $\begin{array}{c}\mathrm{K}_{\mathrm{c}} \cdot 10^{3} \\
\left(\mathrm{kmol} \cdot \mathrm{m}^{-3}\right)\end{array}$ & $\begin{array}{c}\mathrm{D}_{\mathrm{e}} \cdot 10^{4} \\
\left(\mathrm{~m}^{2} \cdot \mathrm{min}^{-1}\right)\end{array}$ \\
\hline 1123 & 7.7 & 5.30 & 3.40 \\
\hline 1148 & 10.6 & 7.77 & 3.20 \\
\hline 1173 & 14.5 & 11.00 & 3,25 \\
\hline 1198 & 19.6 & 15.50 & 2,85 \\
\hline 1223 & 26.0 & 21.40 & 2.90 \\
\hline
\end{tabular}

Table 2. Values proposed by different researchers for the apparent activation energy of the chemical decomposition step.

\begin{tabular}{|c|c|c|}
\hline Researchers & Temperature range (K) & $\mathrm{E}\left(\mathrm{J} \cdot \mathrm{mol}^{-1}\right)$ \\
\hline Fujimoto et al. $[14]$ & $1123-1193$ & 102680 \\
\hline Murthy [19] & $1073-1133$ & 159296 \\
\hline Ingraham and Marier [44] & $1063-1123$ & 169870 \\
\hline Hills [45] & $873-1173$ & 167400 \\
\hline Fuertes et al. [46\} & $1034-1173$ & 176396 \\
\hline Rajeswara Rao [47] & $1010-1148$ & 118000 \\
\hline Feng et al. [48] & $873-1273$ & 115000 \\
\hline Rodriguez et al. [49] & 1150 & 176000 \\
\hline Present work & $1123-1223$ & 175384 \\
\hline Previous paper [40] & $1123-1223$ & \\
\hline
\end{tabular}


Table 3. Values obtained for the total pore volume and apparent porosity of thermally treated samples at different temperatures.

\begin{tabular}{|c|c|c|}
\hline Temperature $(\mathrm{K})$ & Total pore volume $\left(\mathrm{cm}^{3} \cdot \mathrm{g}^{-1}\right)$ & Apparent porosity $(\%)$ \\
\hline 1098 & 0.46 & 56.5 \\
\hline 1123 & 0.46 & 56.6 \\
\hline 1148 & 0.44 & 54.4 \\
\hline 1173 & 0.43 & 54.3 \\
\hline 1198 & 0.40 & 50.4 \\
\hline
\end{tabular}

Table 4. Values of $k, K_{c}$, and $D_{e}$ used in equation (13) and values of $b$ obtained with the best fit $\left(c^{0}{ }_{B}=21.7 \mathrm{kmol} \cdot \mathrm{m}^{-3} ; \mathrm{S}_{\mathrm{sp}}=1354700 \mathrm{~m}^{2} \cdot \mathrm{m}^{-3}\right.$ particle $)$.

\begin{tabular}{|c|c|c|c|c|c|c|c|}
\hline$T(K)$ & $\begin{array}{c}\mathrm{k} \cdot 10^{6} \\
\left(\mathrm{kmol} \cdot \mathrm{m}^{-2} \cdot \mathrm{min}^{-1}\right)\end{array}$ & $\begin{array}{c}K_{c} \cdot 10^{3} \\
\left(\mathrm{kmol} \cdot \mathrm{m}^{-3}\right)\end{array}$ & $\begin{array}{c}D_{e} \cdot 10^{4} \\
\left(m^{2} \cdot \min ^{-1}\right)\end{array}$ & $\begin{array}{c}b \\
100 \% \mathrm{CO}_{2}\end{array}$ & $\begin{array}{c}b \\
70 \% \mathrm{CO}_{2}\end{array}$ & $\begin{array}{c}b \\
53 \% \mathrm{CO}_{2}\end{array}$ & $\begin{array}{c}b \\
38 \% \mathrm{CO}_{2}\end{array}$ \\
\hline 975 & 0.80 & 0.40 & 3.20 & - & - & - & - \\
\hline 1028 & 1.95 & 1.10 & 3.28 & - & - & - & - \\
\hline 1047 & 2.61 & 1.53 & 3.30 & - & - & - & - \\
\hline 1080 & 4.26 & 2.70 & 3.35 & - & - & - & - \\
\hline 1098 & 5.60 & 3.74 & 3.37 & - & - & - & 0.675 \\
\hline 1107 & 6.21 & 4.20 & 3.38 & - & - & 0.693 & - \\
\hline 1113 & 6.74 & 4.59 & 3.40 & - & - & 0.697 & - \\
\hline 1123 & 7.60 & 5.30 & 3.40 & - & 0.715 & 0.700 & 0.685 \\
\hline 1150 & 10.90 & 8.00 & 3.20 & 0.763 & - & - & - \\
\hline 1152 & 11.20 & 8.30 & 3.22 & 0.763 & 0.725 & 0.710 & 0.695 \\
\hline 1155 & 11.40 & 8.56 & 3.23 & 0.765 & - & - & - \\
\hline 1158 & 11.90 & 8.93 & 3.24 & 0.766 & - & - & - \\
\hline 1173 & 14.5 & 11.00 & 3.25 & 0.770 & 0.732 & 0.715 & 0.700 \\
\hline 1195 & 18.9 & 14.9 & 2.85 & 0.780 & 0.740 & 0.725 & 0.710 \\
\hline 1216 & 24.0 & 19.60 & 2.90 & 0.785 & 0.745 & 0.730 & 0.715 \\
\hline
\end{tabular}




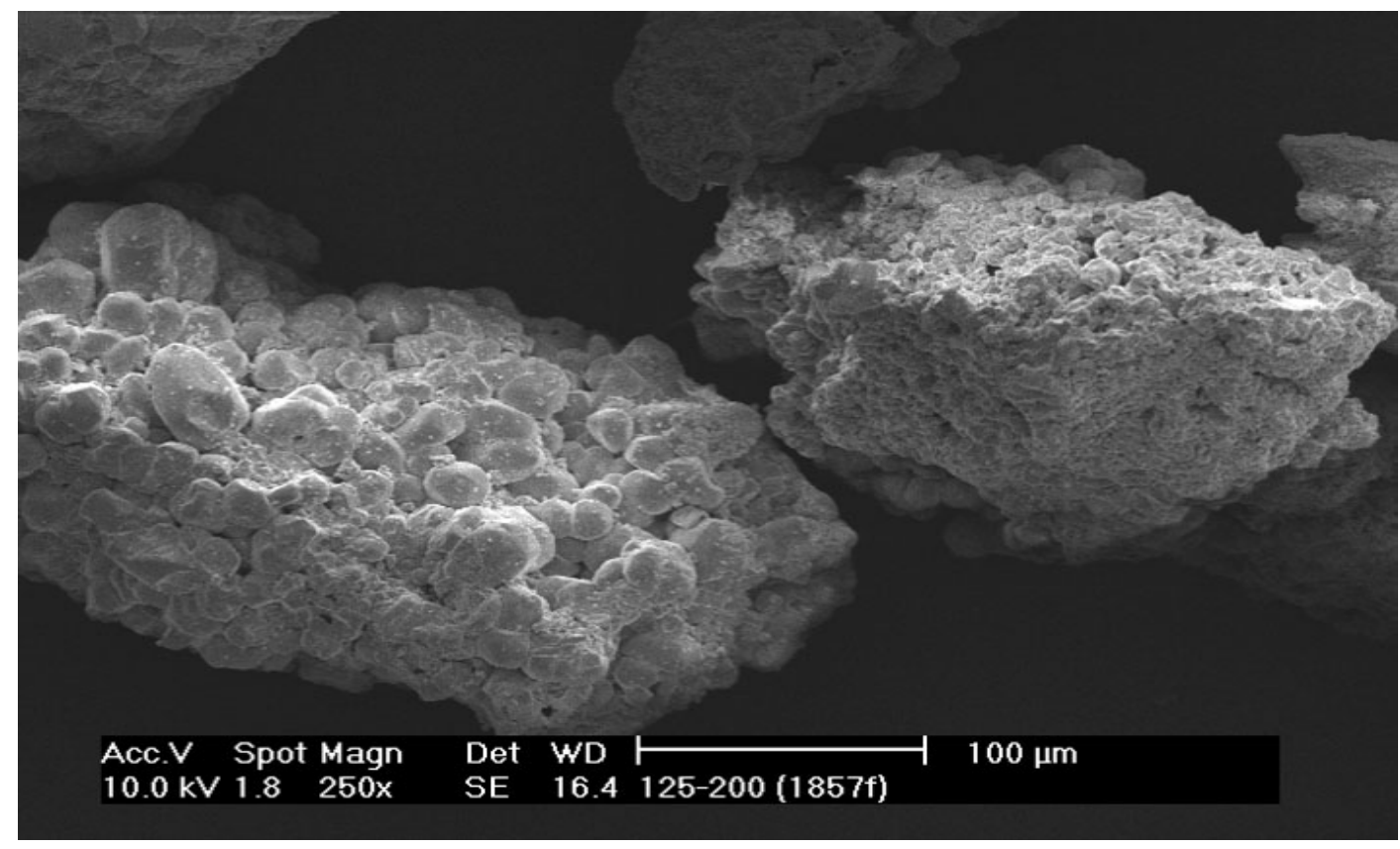

Figure 1. Micrograph of the calcite particles.

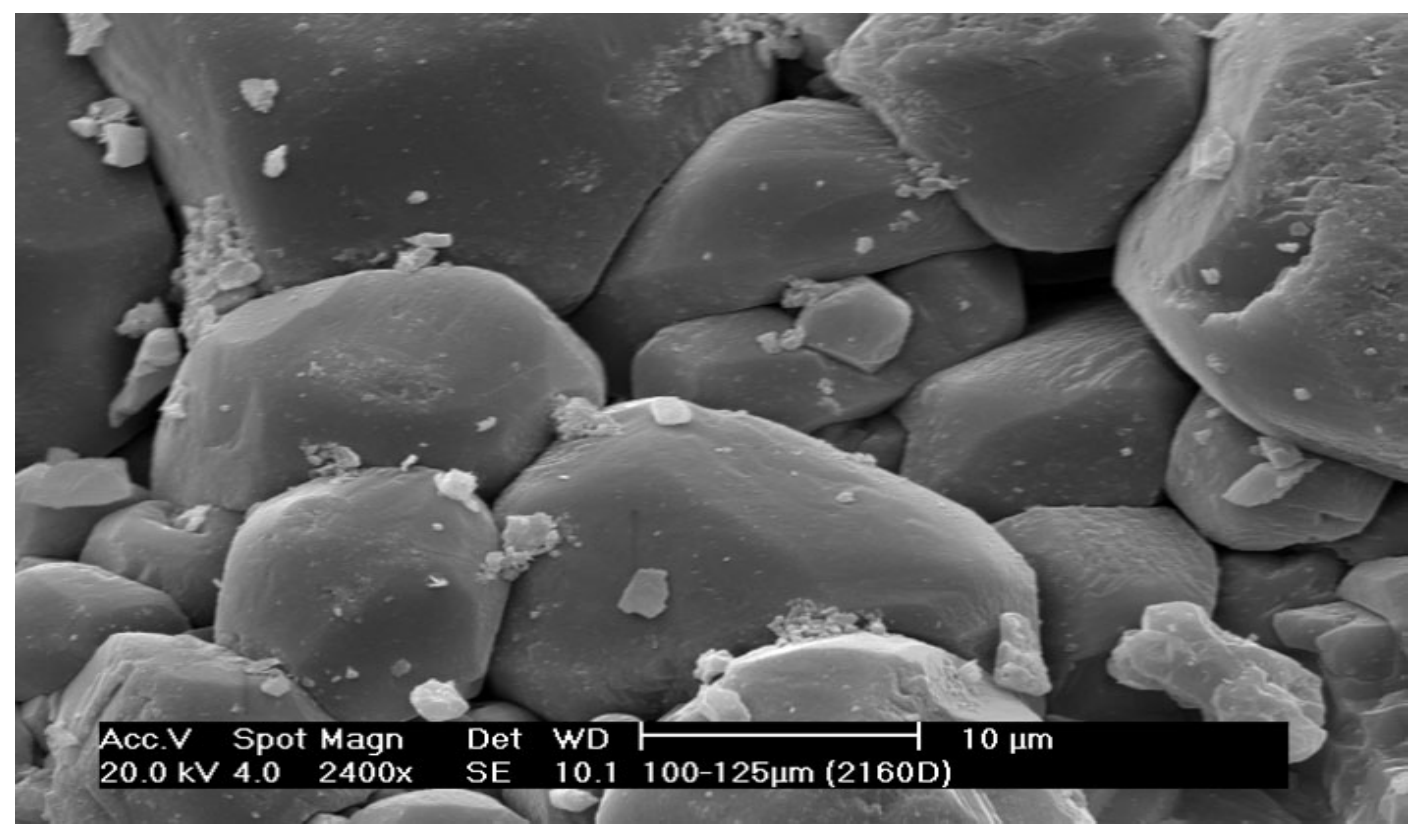

Figure 2. Detail of the microcrystals making up the particle in Figure 1. 


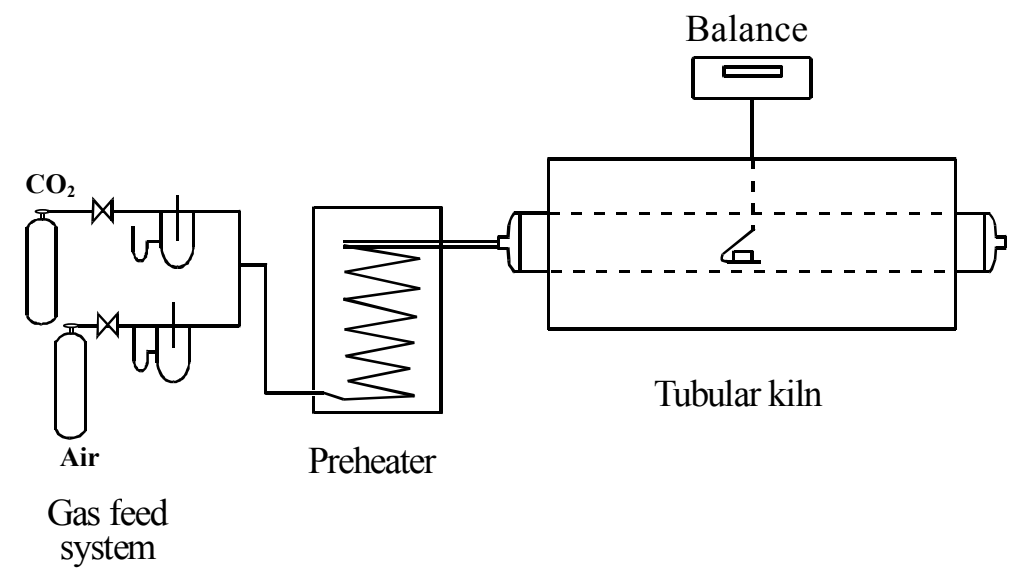

Figure 3. Schematic illustration of the experimental set-up used.

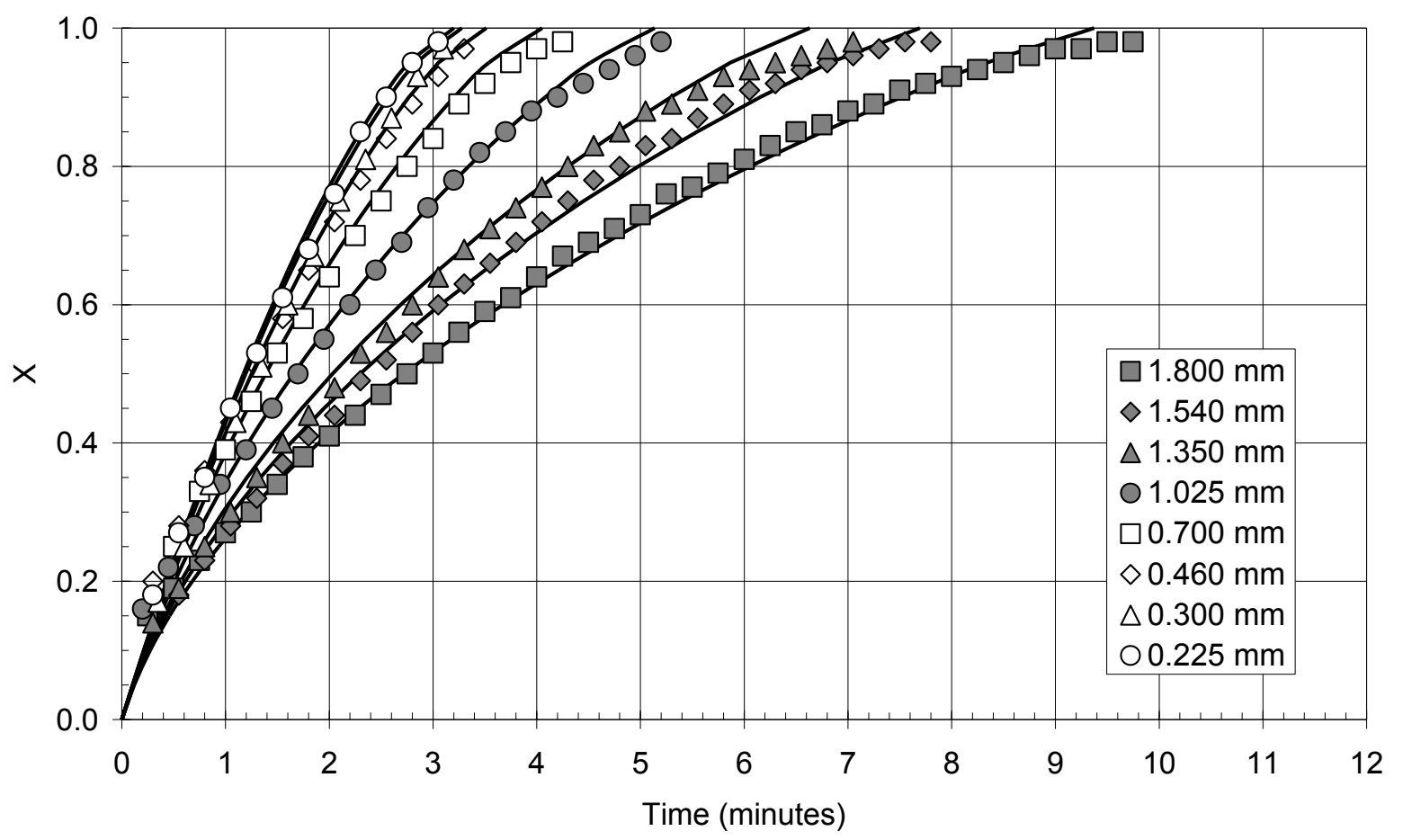

Figure 4. Comparison of the experimental data with the results calculated using equations (9), (10), and (11), in air atmosphere. $T=1123 \mathrm{~K}$. 


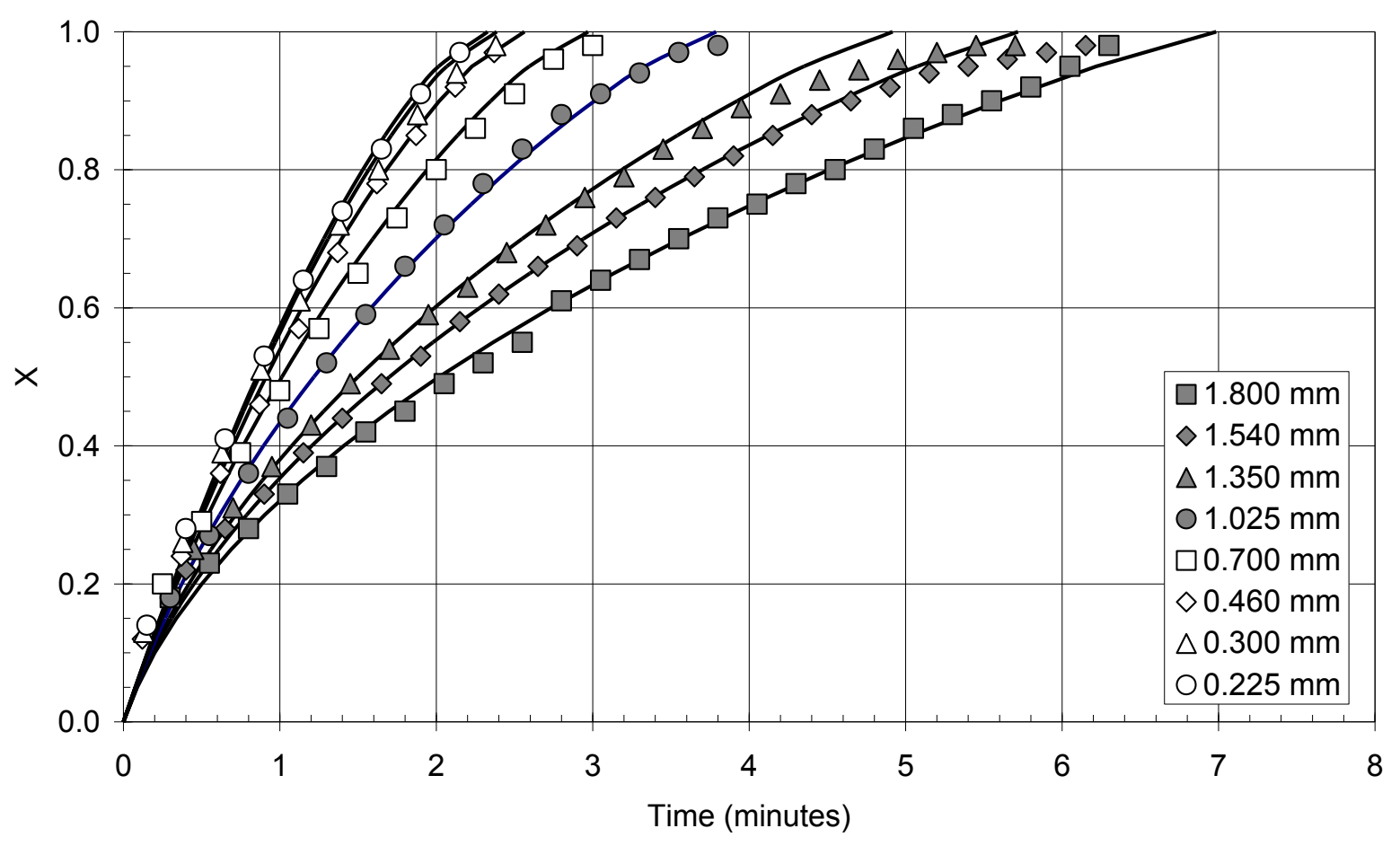

Figure 5. Comparison of the experimental data with the results calculated using equations (9), (10), and (11), in air atmosphere. $T=1148 \mathrm{~K}$.

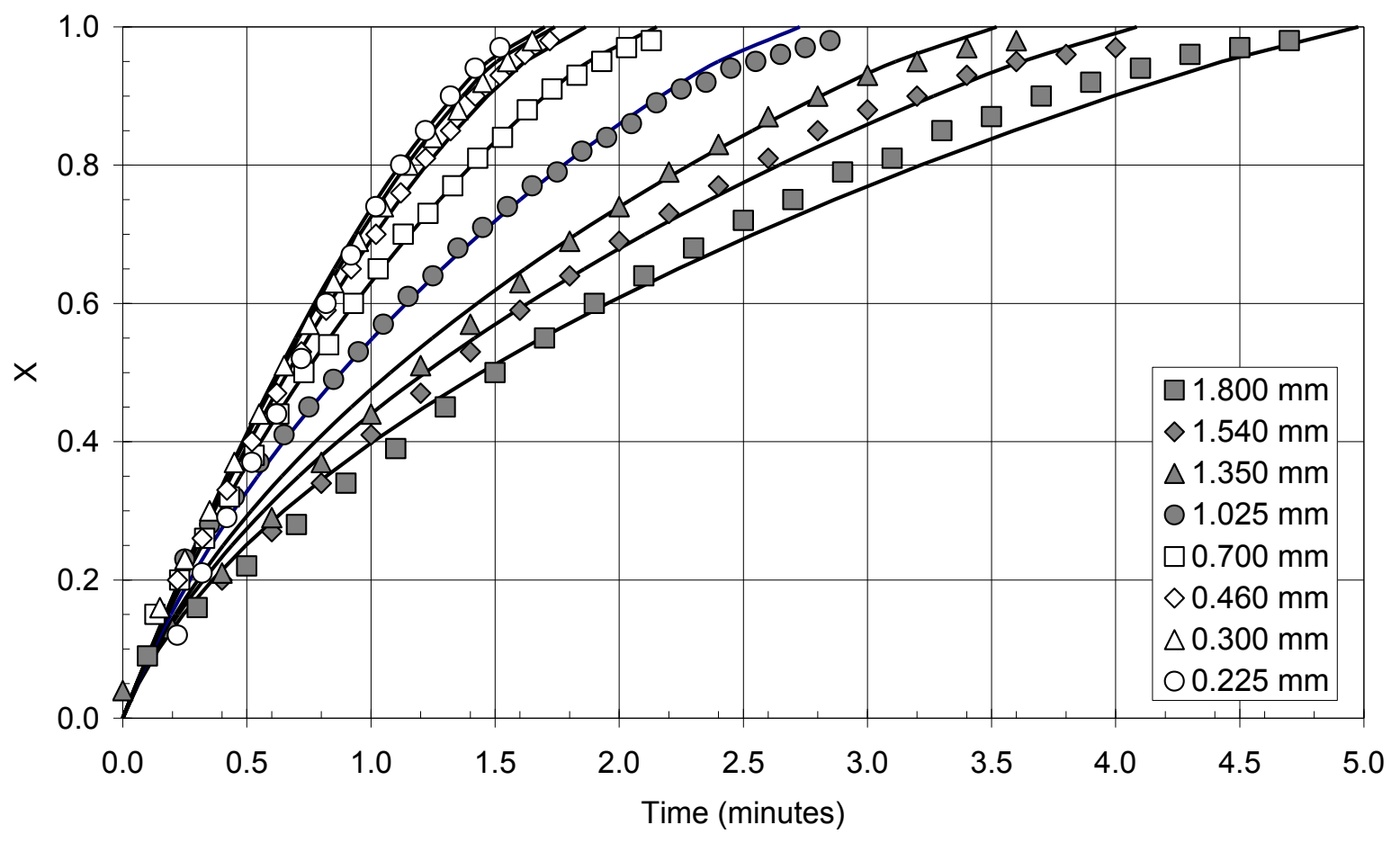

Figure 6. Comparison of the experimental data with the results calculated using equations (9), (10), and (11), in air atmosphere. $T=1173 \mathrm{~K}$. 


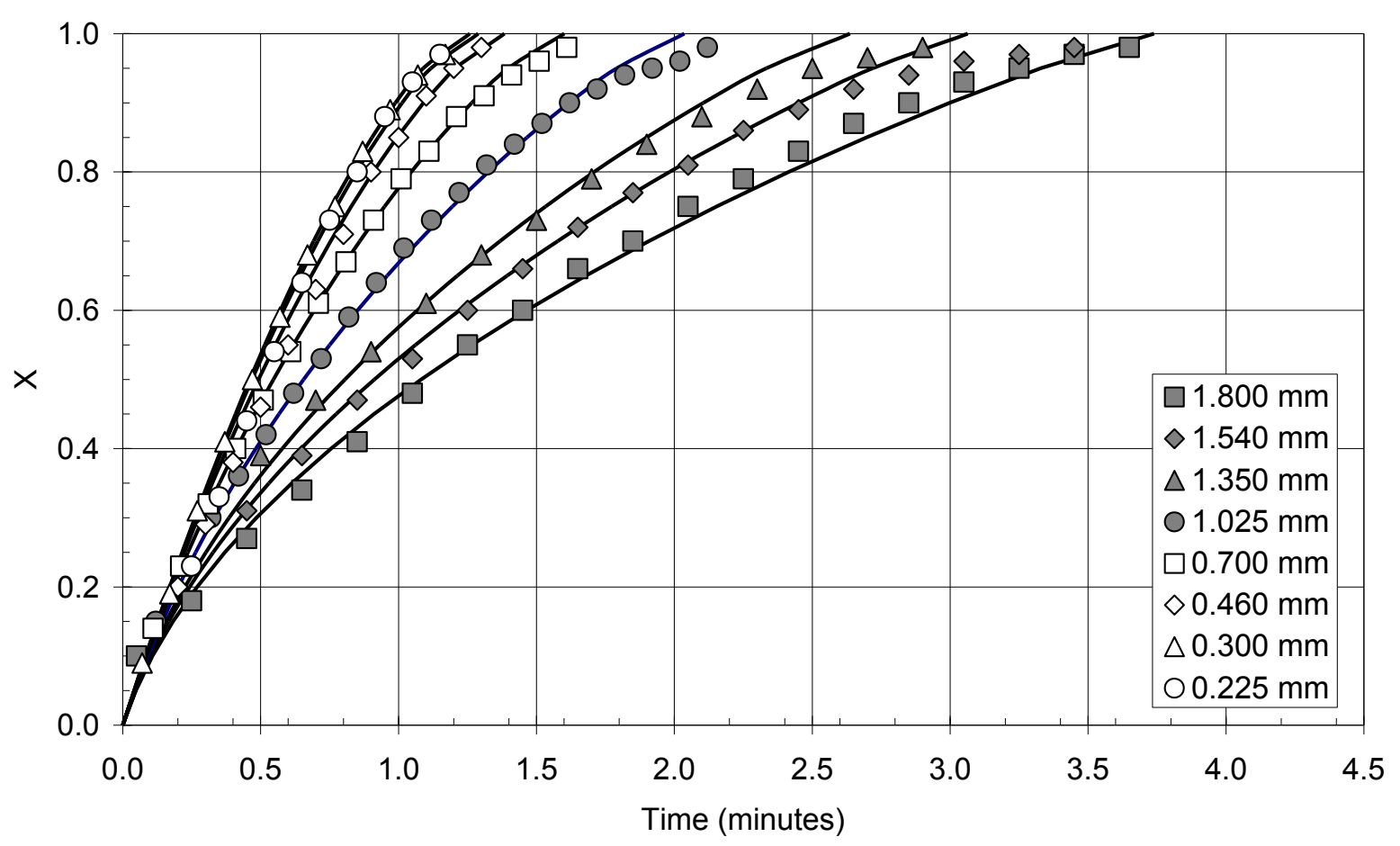

Figure 7. Comparison of the experimental data with the results calculated using equations (9), (10), and (11), in air atmosphere. $T=1198 \mathrm{~K}$.

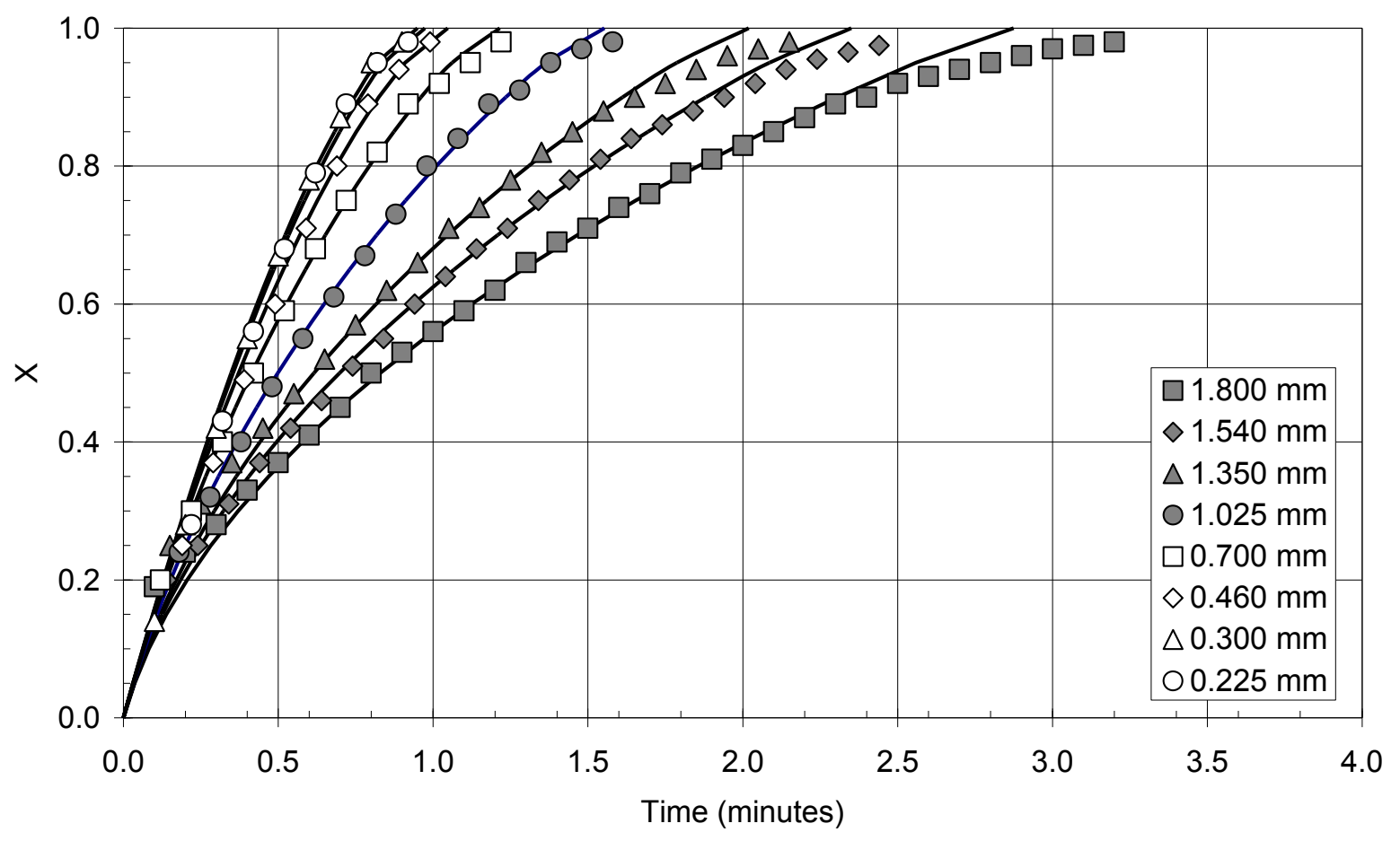

Figure 8. Comparison of the experimental data with the results calculated using equations (9), (10), and (11), in air atmosphere. $T=1223 \mathrm{~K}$. 


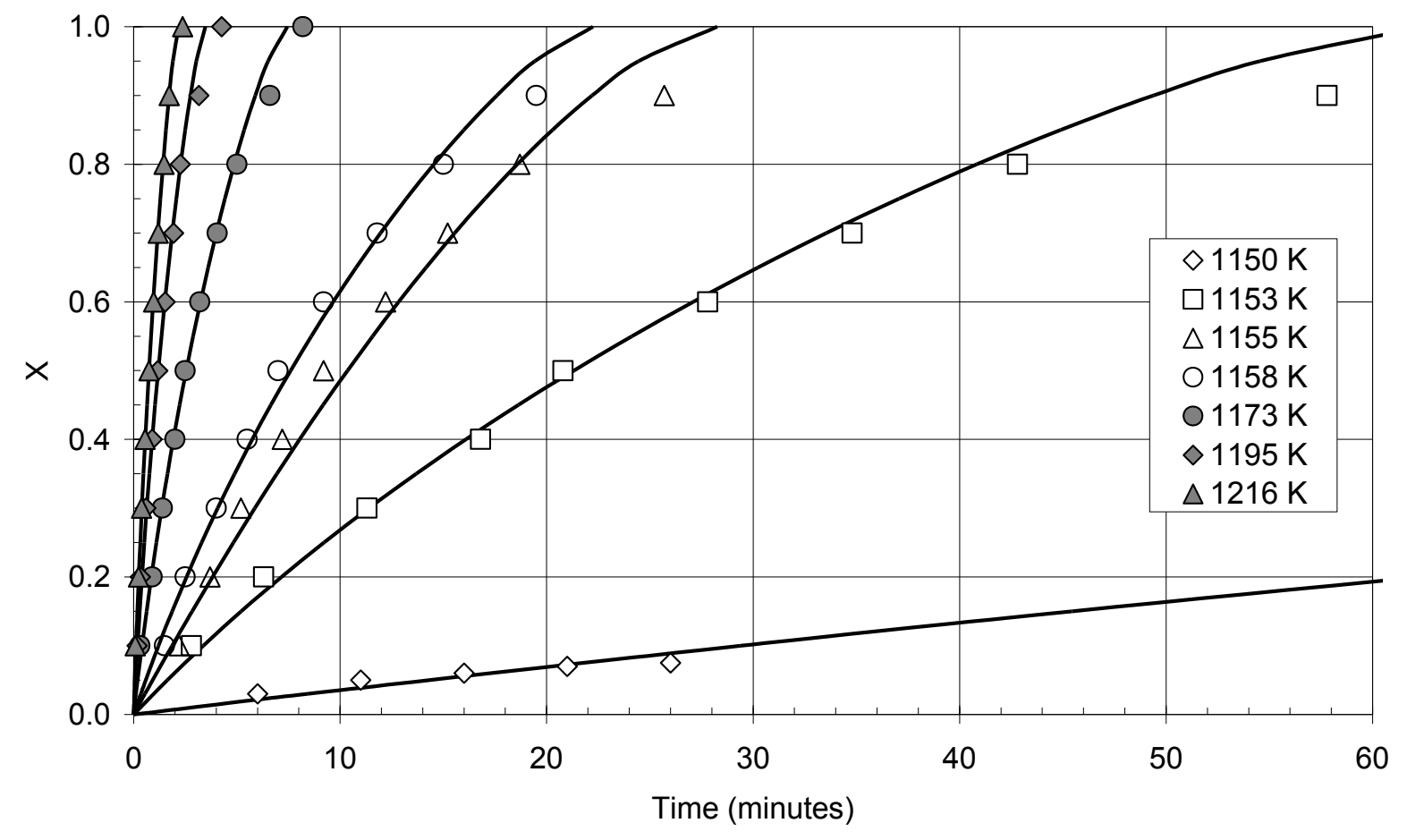

Figure 9. Comparison of the experimental data with the results calculated using equations (13), (10), and (11), in $\mathrm{CO}_{2}$ atmosphere. $r_{S}=0.7 \mathrm{~mm}$.

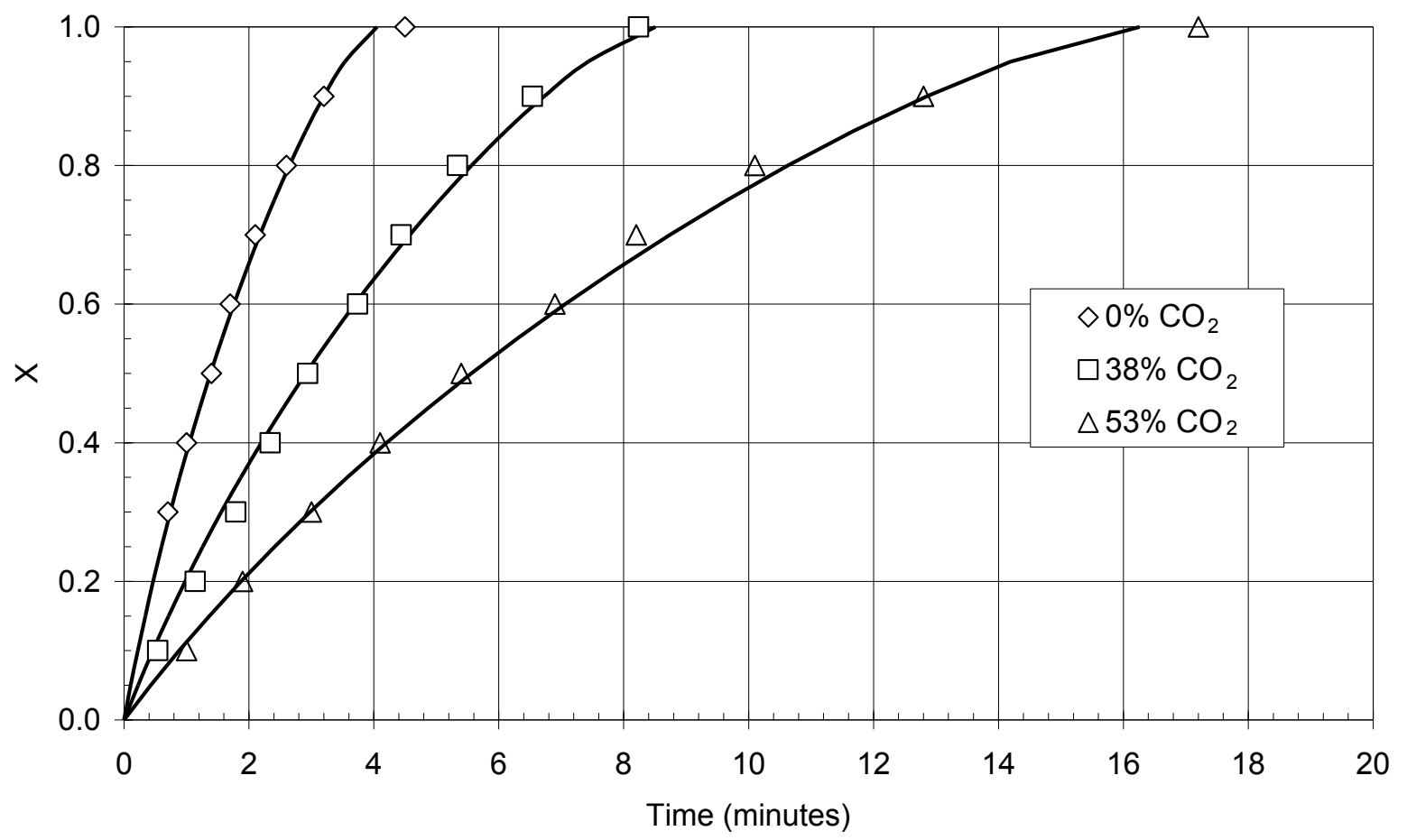

Figure 10. Comparison of the experimental data with the results calculated using equations (13), (10), and (11), in different concentrations of air and $\mathrm{CO}_{2} \cdot T=1123 \mathrm{~K} . r_{S}=$ 
$0.7 \mathrm{~mm}$

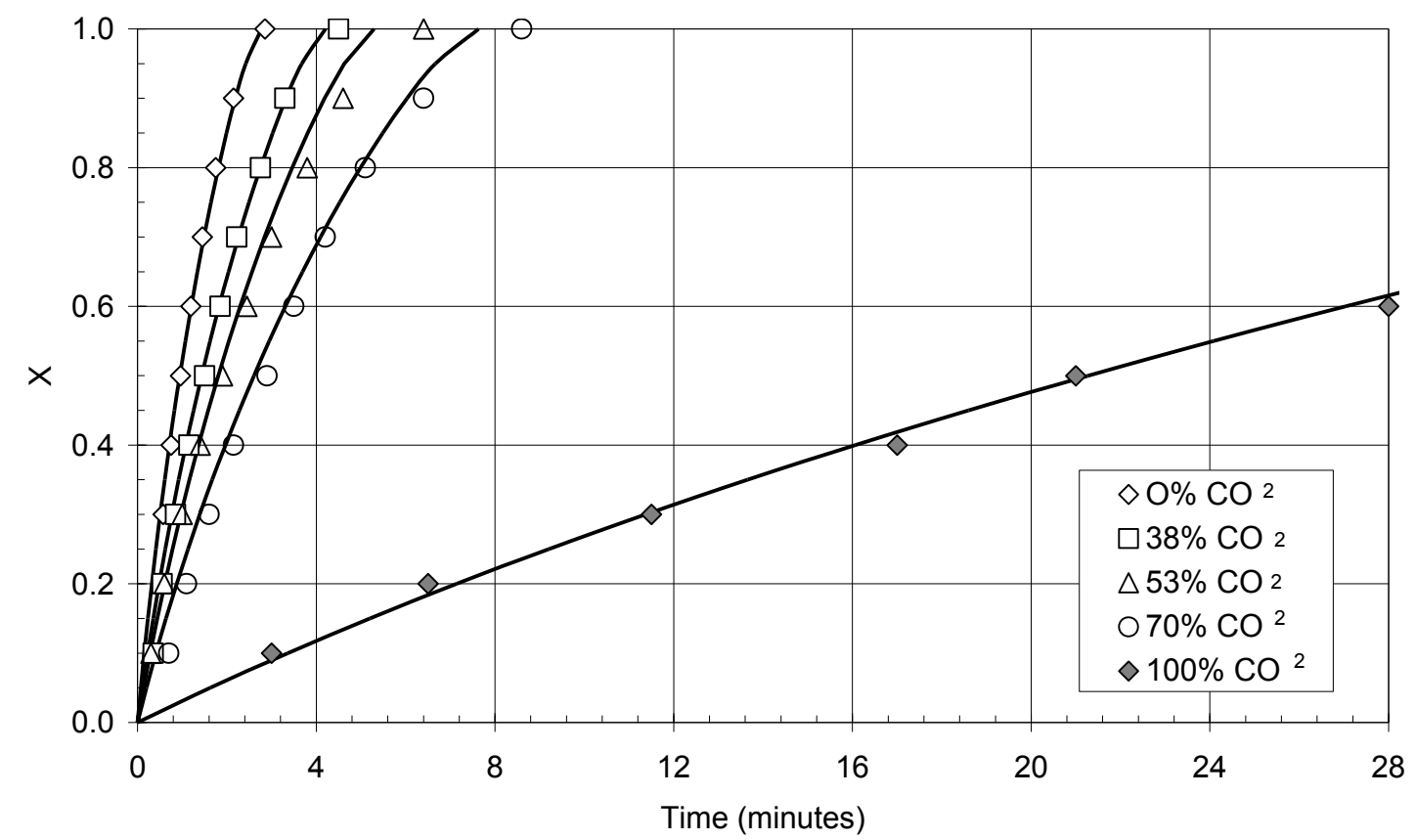

Figure 11. Comparison of the experimental data with the results calculated using equations (13), (10), and (11), in different concentrations of air and $\mathrm{CO}_{2} . T=1153 \mathrm{~K} . r_{S}=$ $0.7 \mathrm{~mm}$.

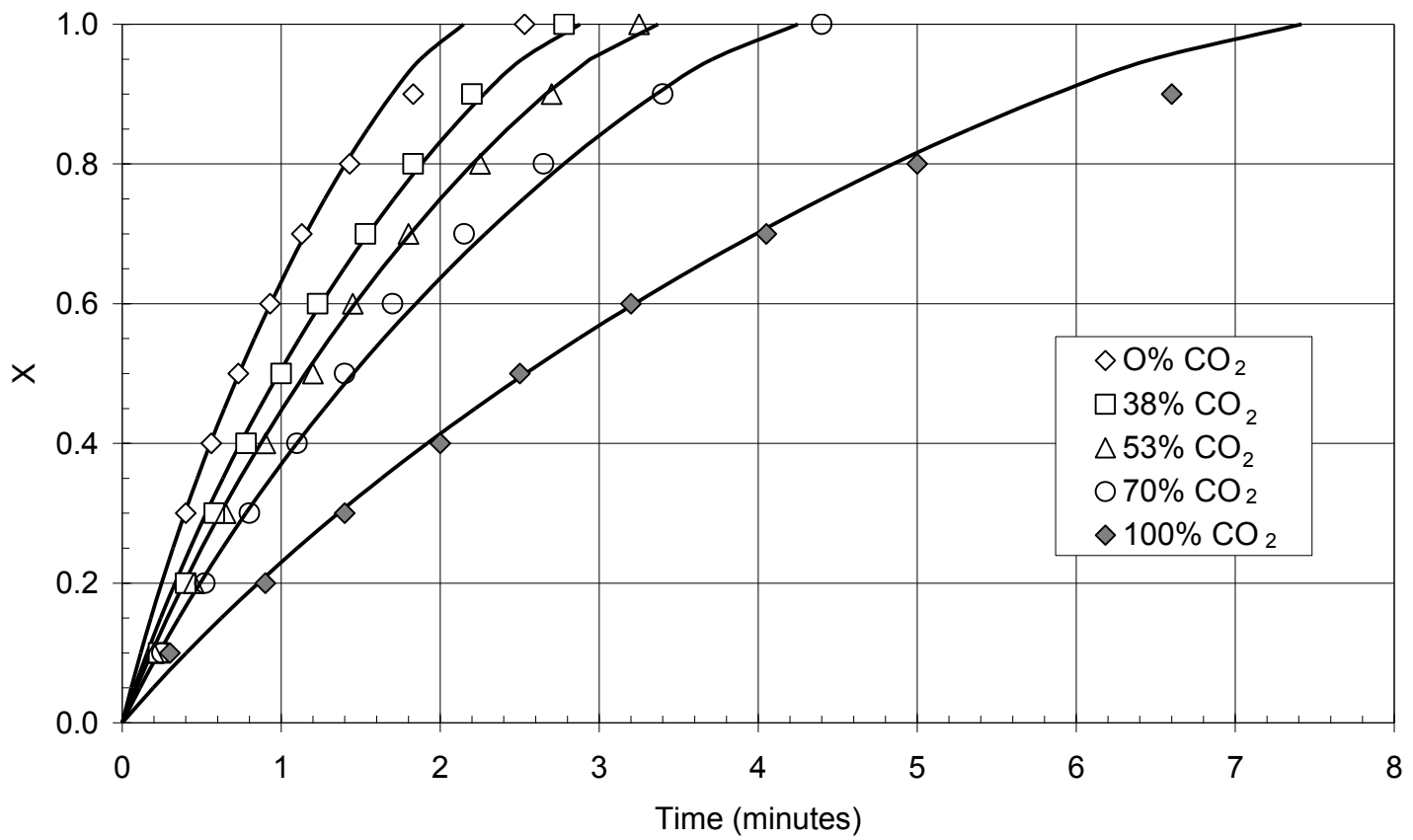

Figure 12. Comparison of the experimental data with the results calculated using equations (13), (10), and (11), in different concentrations of air and $\mathrm{CO}_{2} . T=1173 \mathrm{~K} . r_{S}=$ $0.7 \mathrm{~mm}$. 


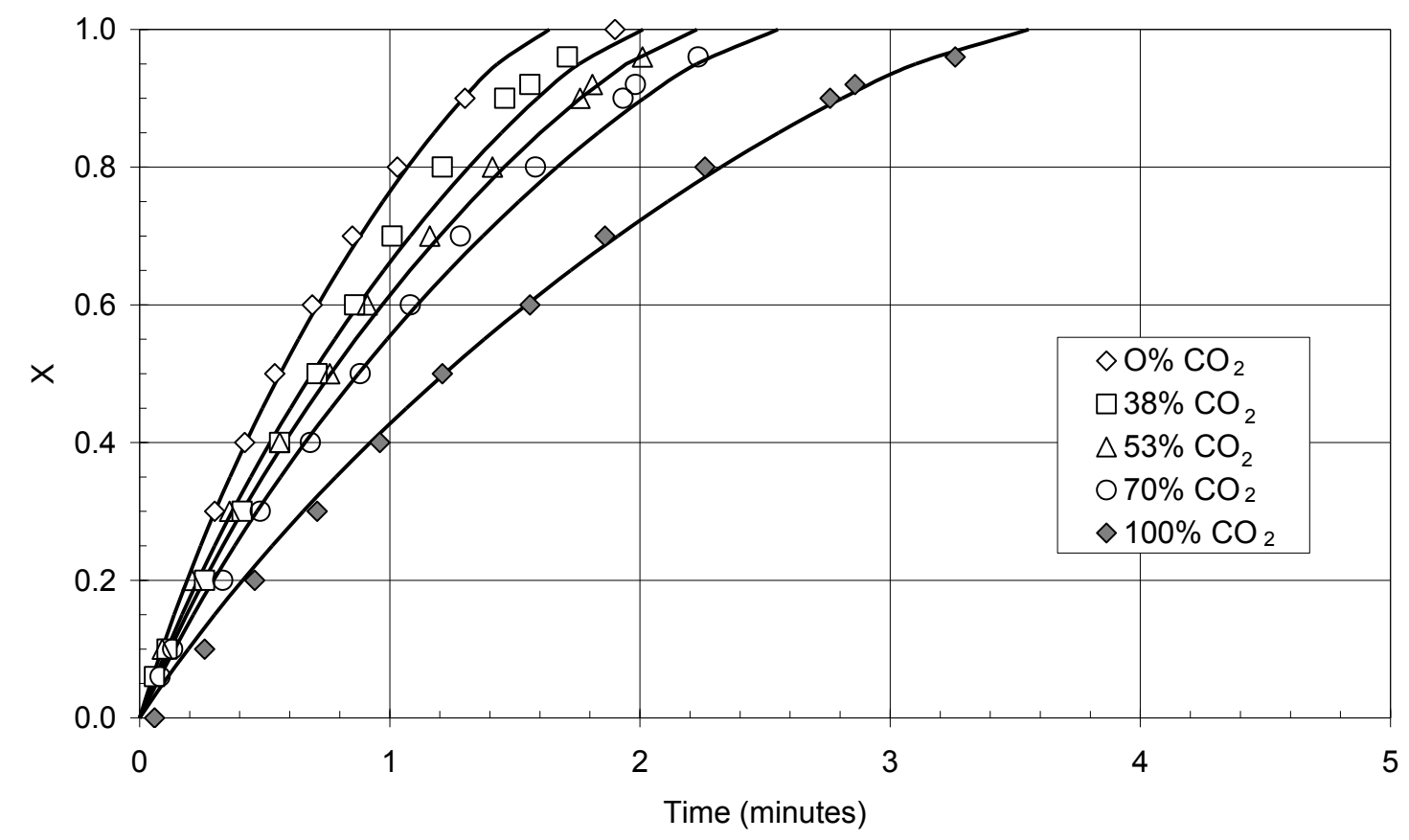

Figure 13. Comparison of the experimental data with the results calculated using equations (13), (10), and (11), in different concentrations of air and $\mathrm{CO}_{2} . T=1195 \mathrm{~K} . r_{S}=$ $0.7 \mathrm{~mm}$

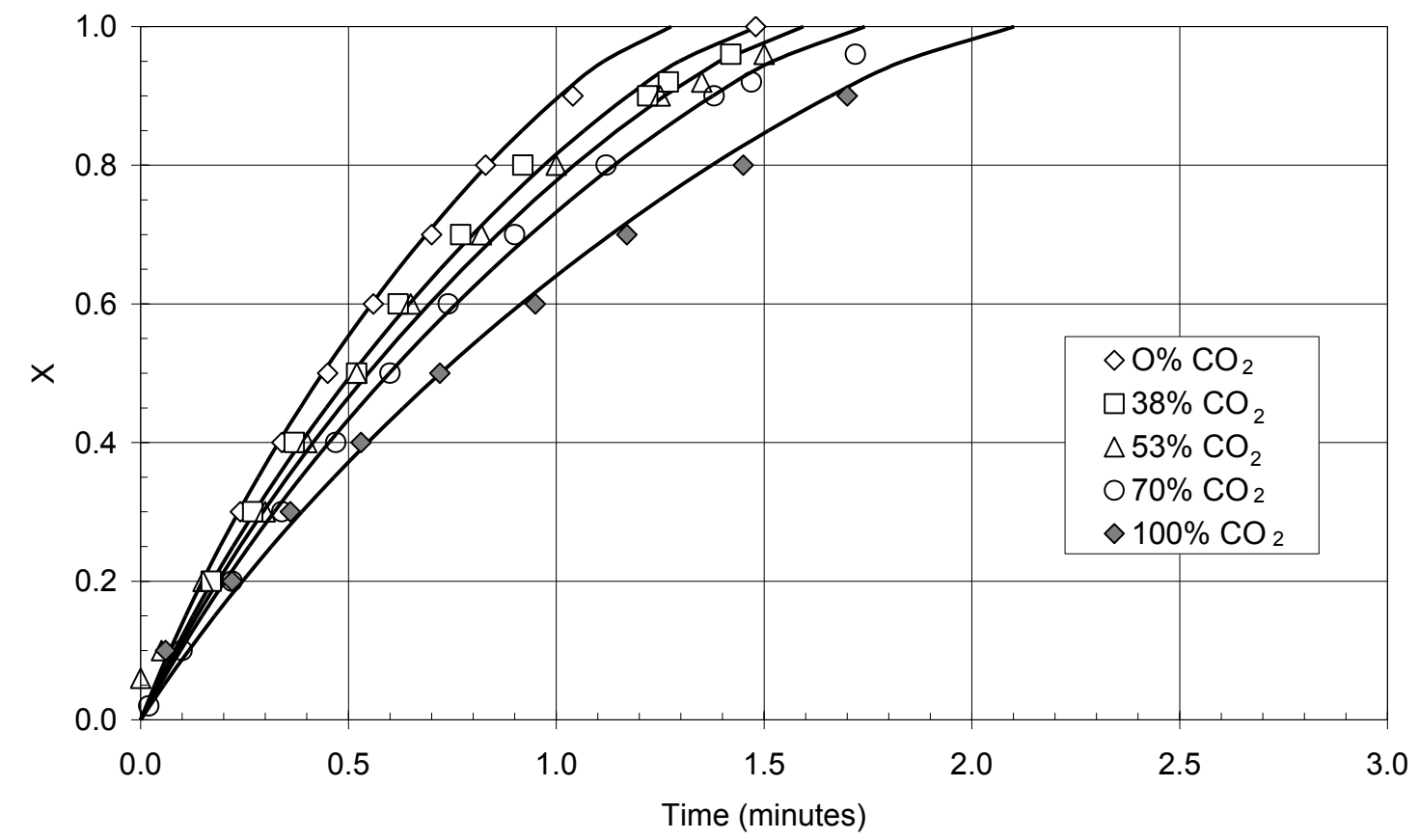

Figure 14. Comparison of the experimental data with the results calculated using equations (13), (10), and (11), in different concentrations of air and $\mathrm{CO}_{2} \cdot T=1216 \mathrm{~K} . r_{S}=$ $0.7 \mathrm{~mm}$ 


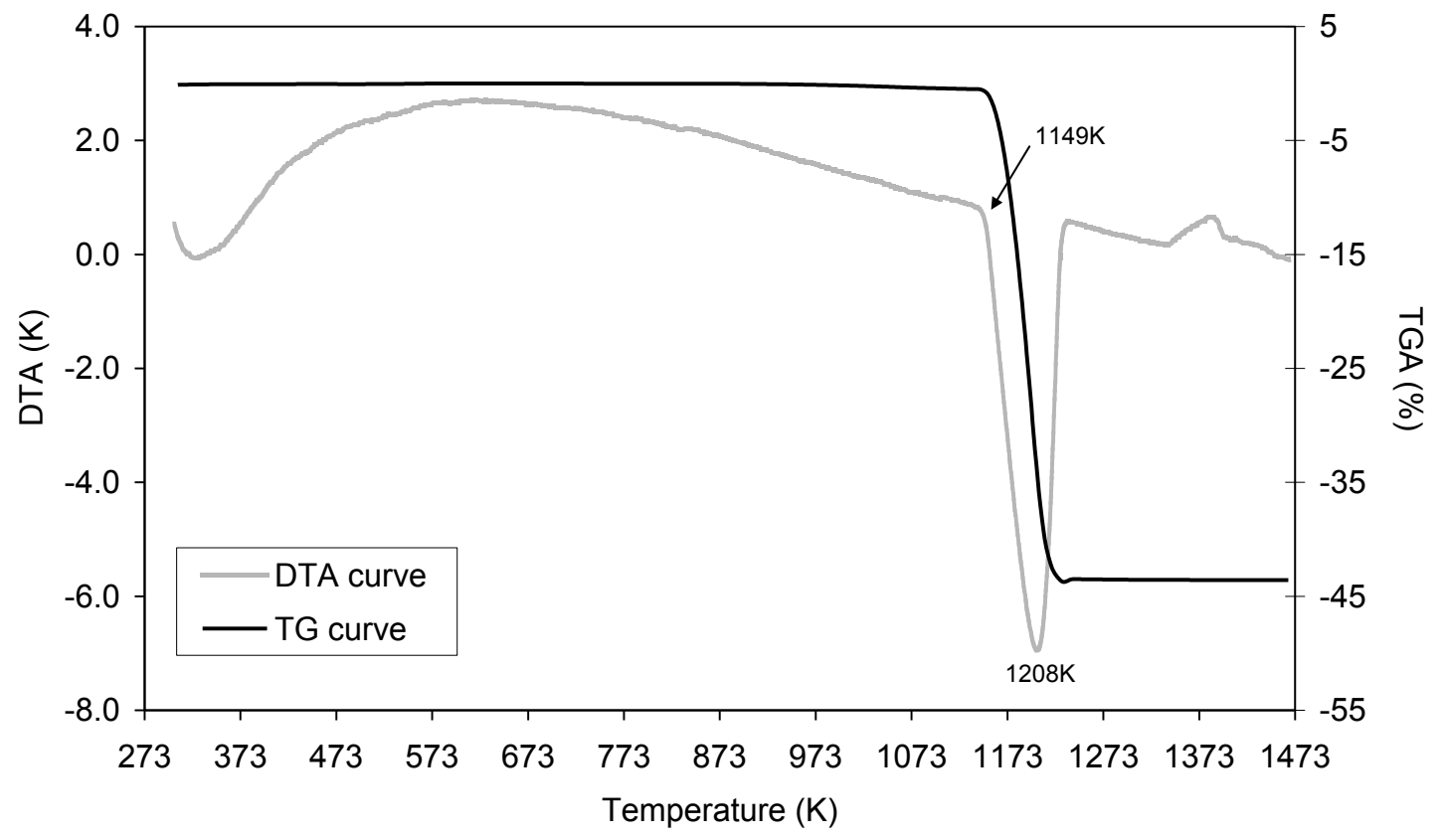

Figure 15. DTA-TGA diagram of the calcite particles in $\mathrm{CO}_{2}$ atmosphere.

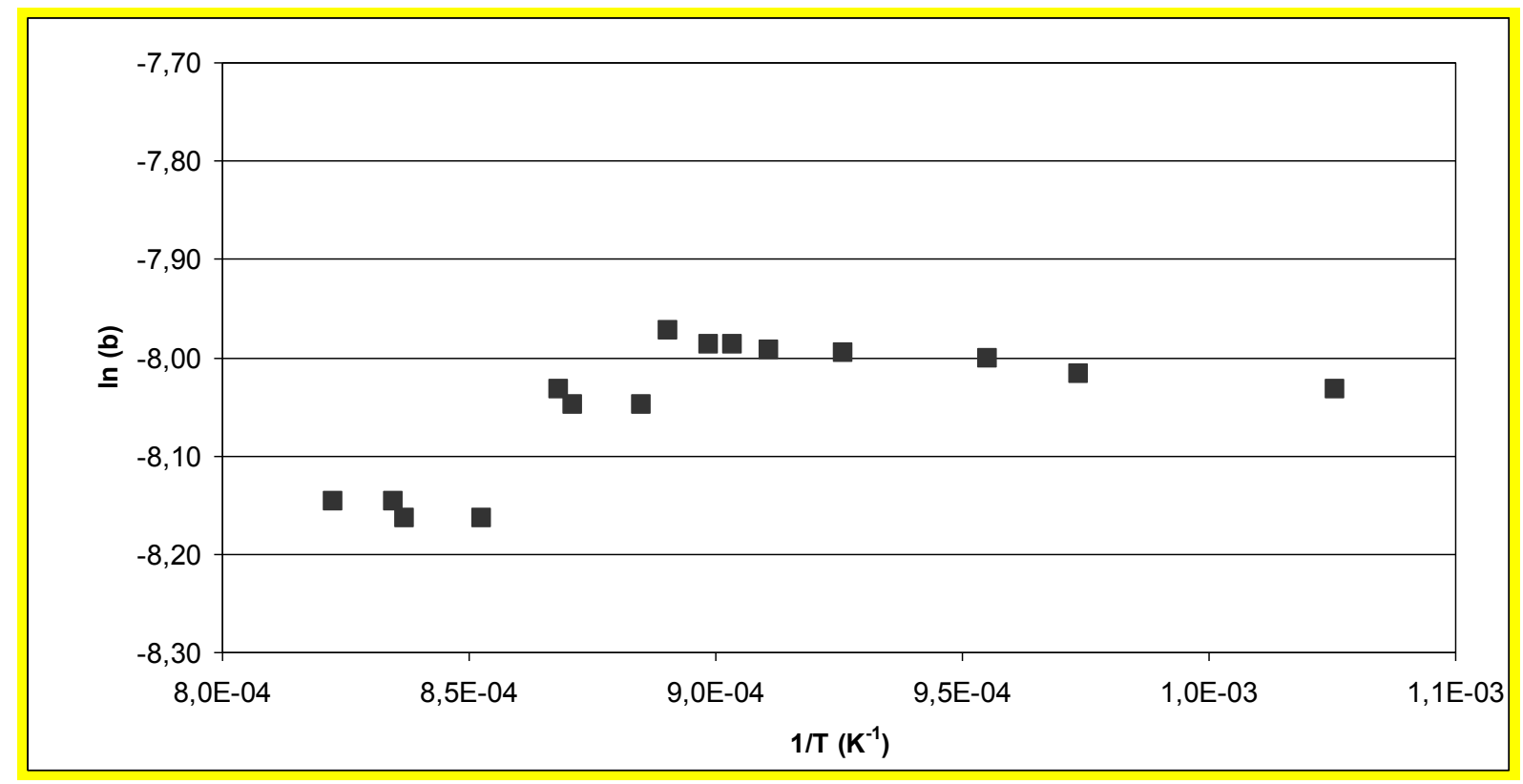

Figure 16. Variation of effective diffusivity $\left(D_{e}\right)$ with temperature $(K)$. 


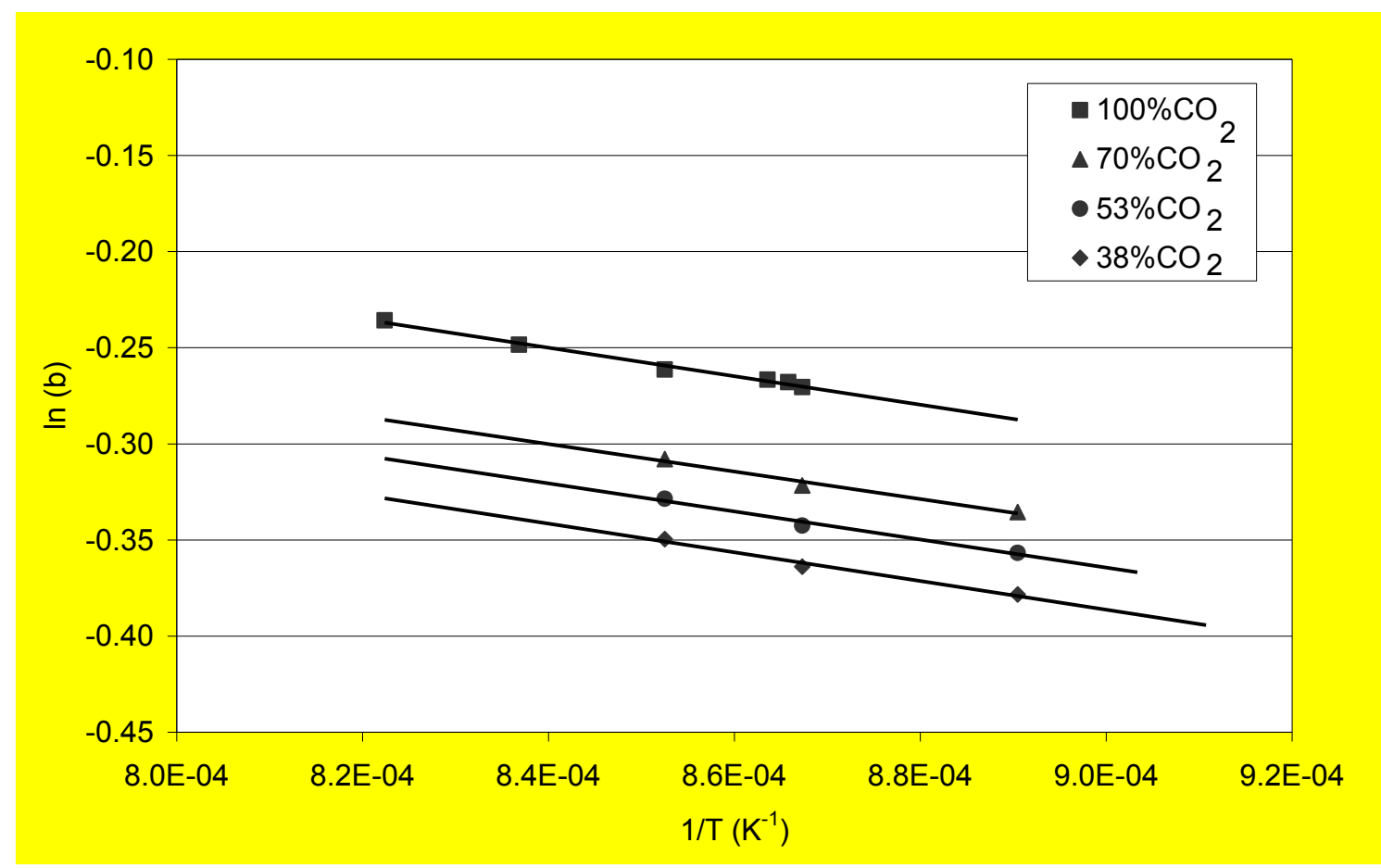

Figure 17. Variation of parameter $b$ with temperature $(\mathrm{K})$ and $\mathrm{CO}_{2}$ percentage

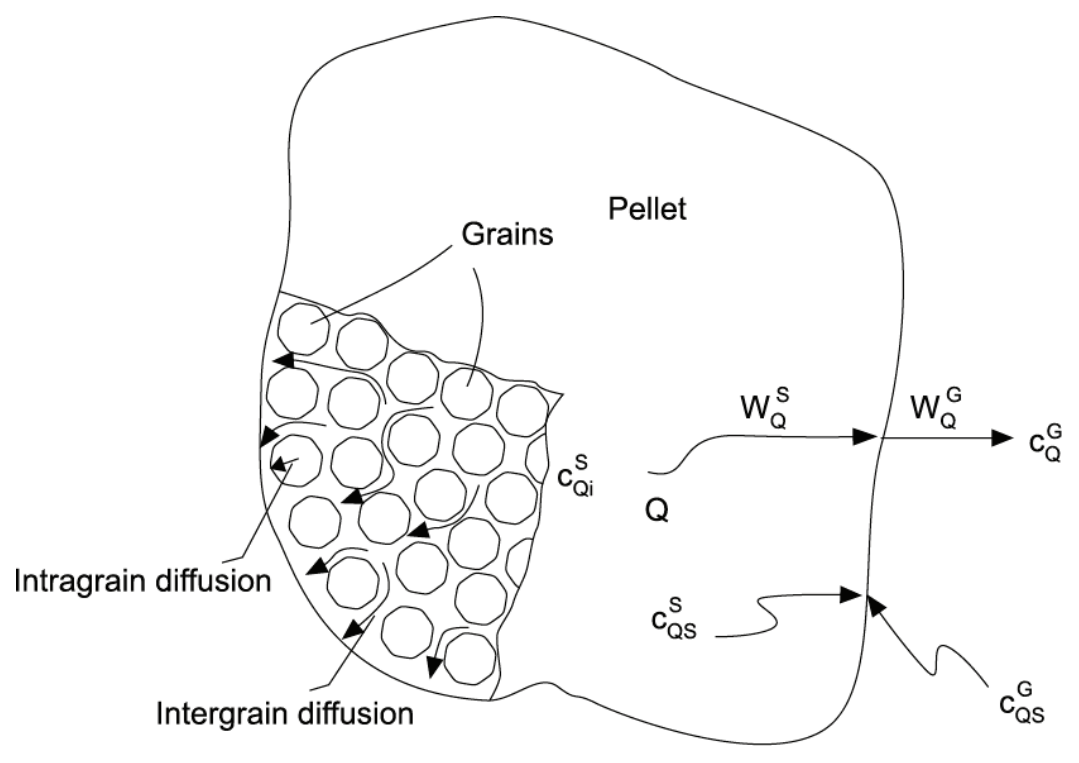

Figure A.1. Scheme of the kinetic model considered. 